\title{
A Multi-Disaster-Scenario Distributionally Robust Planning Model for Enhancing the Resilience of Distribution Systems
}

\author{
Gang Zhanga , Feng Zhang ${ }^{* a}$, Xin Zhang ${ }^{\mathrm{b}}$, Qiuwei Wu ${ }^{\mathrm{a}, \mathrm{c}}$, Ke Meng ${ }^{\mathrm{d}}$ \\ ${ }^{a}$ Key Laboratory of Power System Intelligent Dispatch and Control of Ministry of Education, Shandong \\ University, No.17923, Jingshi Road, Ji'nan 250061, China \\ ${ }^{b}$ The Energy and Power Theme, School of Water, Energy and Environment, Cranfield University, Cranfield \\ MK43 OAL, U.K. \\ ${ }^{c}$ Center for Electric Power and Energy (CEE), Department of Electrical Engineering, Technical University of \\ Denmark (DTU), 2800, Kgs. Lyngby, Denmark \\ ${ }^{d}$ School of Electrical Engineering and Telecommunications, The University of New South Wales, NSW 2052,
}

Australia

Corresponding author: Tel.: +86 531-81696119. E-mail address: fengzhang@ sdu.edu.cn

\begin{abstract}
Resilience oriented network planning provides an effective solution to protect the distribution system from natural disasters by the pre-planned line hardening and backup generator allocation. In this paper, a multidisaster-scenario based distributionally robust planning model (MDS-DRM) is proposed to hedge against two types of natural disaster-related uncertainties: random offensive resources (ORs) of various natural disasters, and random probability distribution of line outages (PDLO) that are incurred by a certain natural disaster. The OR uncertainty is represented by the defined probability-weighted scenarios with stochastic programming, and the PDLO uncertainty is modeled as the moment based ambiguity sets. Moreover, the disaster recovery strategies of network reconfiguration and microgrid formation are integrated into the pre-disaster network planning for resilience enhancement in both planning and operation stages. Then, a novel primal cut based decomposition solution method is proposed to improve the computational efficiency of the proposed model. In particular, the equivalent reformulation of the original MDS-DRM is first derived to eliminate the PDLOrelated variables. Then, the reformulation problem is solved by the proposed primal cut based decomposition method and linearization techniques. Finally, Simulation results are demonstrated for IEEE 13-node, 33-node and 135-node distribution systems to validate the effectiveness of the proposed method in enhancing the disaster-induced network resilience.
\end{abstract}

Keywords: Distributionally robust method; resilience; network planning; power distribution system; stochastic programming; uncertainty.

\section{NOMENCLATURE}

\begin{tabular}{|c|c|c|c|}
\hline 1) Sets & ectors, and Indices & $s$ & Index for natural disaster scenarios \\
\hline & Indices for buses & $t$ & Index of time periods \\
\hline$k, \omega$ & Index for iterations of Algorithm 1 and 2 & $\Phi^{s}$ & The ambiguity set for the distribution $\Pi^{s}$ \\
\hline
\end{tabular}


$n(i), m(i)$ Sets of all parent buses and children buses of bus $i$

$\Pi^{s}\left(Z^{s}\right) \quad$ Set of all probability distributions on $Z^{s}$

$S \quad$ Set of natural disaster scenarios

$T \quad$ Set of time periods

$V^{s} \quad$ Set of binary variables in scenario $s$ in Algorithm 2

$\Psi \quad$ The feasibility set of the planning decisions

$Z^{s} \quad$ Set of damaged lines in scenario $s$

$\phi^{s} \quad$ Set of damaged lines due to scenario $s$ used in Algorithm 1

$\Omega_{B} \quad$ Set of branches

$\Omega_{N} \quad$ Set of Nodes

$\mathbf{a}, \mathbf{b}, \mathbf{c}, \quad$ Vectors of constants used in model d, e abbreviation

$\mathbf{A}, \mathbf{B}, \mathbf{C}$, Matrixes of coefficients used in model

D, E, F abbreviation

$\mathbf{x}^{\mathrm{s}} \quad$ Vector of continuous variable in the operational stage after the scenario $s$

$\mathbf{y} \quad$ Concatenation vector of planning decision variables $y_{i j}^{h}$ and $y_{i}^{g}$

$\mathbf{z}^{s} \quad$ Vector of attacker decision variable $z_{i j}^{s}$

$\mathbf{v}^{\mathbf{s}} \quad$ Vector of binary variables in the operational stage after the scenario $s$

$\alpha^{s}, \lambda^{s} \quad$ Vector of dual variables of the constraint in the scenario $\mathrm{s}$

\section{2) Parameters}

$c_{i}^{g} \quad$ Annual capital cost for placing B-DGs at bus $i$

$c_{i j}^{h} \quad$ Annual capital cost for hardening line $i j$

$c_{i}^{l} \quad$ Penalty costs for load shedding at bus $i$

$G$ Budgets for the number of B-DG installation

$H \quad$ Budget for the number of hardening lines

$K^{a v e, s} \quad$ The expected number of the lines damaged by natural disaster $s$

$K^{s} \quad$ The maximum number of damaged lines in scenario $s$

M The big number used in model formulation and linearization

$N_{\text {bus }} \quad$ The total number of buses

$p_{i j}^{F I X, s} \quad$ The estimated PDLO in scenario $s$, which is invariable in stochastic method

$p_{i j}^{L, s} \quad$ The lower bound of the outage probability

of line $i j$ in scenario $s$

$P_{i t}^{L}, Q_{i t}^{L} \quad$ Active and reactive load at bus $i$ and time $t$

$P_{i}^{\max }, Q_{i}^{\max }$ Active and reactive power limits of B-DG at bus $i$

$P_{i j}^{\max }, Q_{i j}^{\max }$ Active and reactive power limits of line $i j$

$\Pi^{s} \quad$ The probability distribution of $\mathbf{z}^{s}$ in scenario $p_{i j}^{U, s} \quad$ The upper bound of the outage probability of line $i j$ in scenario $s$

$R_{i j}, X_{i j} \quad$ The resistance and reactance of line $i j$

$U_{0} \quad$ The reference voltage magnitude

$U_{i}^{\text {max }}, U_{i}^{\text {min }}$ Maximum and minimum voltage magnitudes of bus $i$

$\rho^{s} \quad$ Occurrence probability of scenario $s$

\section{3) Variables}

$F_{i j}^{s} \quad$ The fictitious flow of line $i j$ in the scenario

$P_{i t}^{g, s}, Q_{i t}^{g, s} \quad$ Active and reactive power output of DGs at bus $i$ and time $t$ in the scenario $s$

$P_{i j, t}^{s}, Q_{i j, t}^{s} \quad$ Active and reactive power flow of line $i j$ at time $t$ in the scenario $s$

$\Delta P_{i t}^{s} \quad$ Active load shedding at bus $i$ at time $t$ in the disaster scenario $s$

$\Delta Q_{i t}^{s} \quad$ Reactive load shedding at bus $i$ and time $t$ in the scenario $s$

$U_{i t}^{s} \quad$ Voltage amplitude at bus $i$ and time $t$ in the scenario $s$

$v_{i}^{c 1, s}, v_{i}^{c 2, s} \quad$ Binary variables used in model linearization

$v_{i j}^{d, s} \quad$ Binary variable, with the value of 1 if line $i j$ is either hardened or not damaged in the scenario $s$, and 0 otherwise

$v_{i}^{e, s} \quad$ The binary variable, the value is 1 if bus $i$ is at one end of faulted lines in the scenario $s$, and 0 otherwise

$v_{i j}^{q, s} \quad$ Binary variable, the value of 1 if the final status of line $i j$ is normal in the scenario $s$, and 0 otherwise

$v_{i}^{r, s} \quad$ Binary variable, the value is 1 if bus $i$ is chosen as the root bus in the scenario $s$, and 0 otherwise

$v_{i j}^{w, s} \quad$ Binary variable, the value is 1 if the switch equipped in line $i j$ is closed in the scenario $s$, and 0 otherwise

$y_{i}^{g} \quad$ Binary variable, the value of 1 if a new BDG is placed at bus $i$, and 0 otherwise;

$y_{i j}^{h} \quad$ Binary variable, the value of 1 if line $i j$ is hardened, and 0 otherwise

$z_{i j}^{s} \quad$ Binary variable, the value is 0 if line $i j$ is damaged in scenario $s$, and 1 otherwise;

$\beta^{s} \quad$ Dual variable of the constraint in the scenario $s$

$\mu_{i j}^{s} \quad$ Supplementary variable used in model linearization

\section{4) Abbreviations}

B-DG Backup distributed generator

CCG Column-and-Constraint Generation 


$\begin{array}{llll}\text { CRM } & \text { Conventional robust model } & \text { PDLO } & \text { Probability distribution of line outage } \\ \text { DRM } & \text { Distributionally robust model } & \text { PH } & \text { Progressive hedging } \\ \text { EEV } & \text { Expected value of the EV solution } & \text { RL-CPM } & \text { Reliability constrained planning model } \\ \text { EV } & \text { Expected value solution } & \text { RP } & \text { Recourse problem solution } \\ \text { MDS-DRM } & \begin{array}{l}\text { Multi-disaster-scenario distributionally } \\ \text { robust planning model }\end{array} & \text { RS-OPM } & \text { Resilience oriented planning model } \\ \text { MILP } & \text { Mixed integer linear programming } & \text { WS } & \text { Wait-and-see solution } \\ \text { OR } & \text { Offensive resource } & & \end{array}$

\section{Introduction}

Modern power distribution systems are vulnerable to natural disasters due to their environmentally exposed assets and infrastructure [1]. In 2008, a snow storm of Southern China caused $124,00035 \mathrm{kV}$ and $10 \mathrm{kV}$ distribution line outages according to the 2008 statistics report of the State Grid. In 2012, hurricane Sandy landed on the east coast of the United States and damaged nearly 900 transformers and 1000 poles. In addition, weatherrelated outages in distribution systems will directly affect the continuity of power services to customers [2]. To mitigate power outages, the concept of power system resilience was introduced to highlight the necessity and develop strategies to protect the distribution systems against natural disasters [3].

Resilience is defined as the capability of power distribution system for anticipation, absorption and quickly recovery from extreme events, such as natural disasters [4]. Distribution network planning has been proved to be an efficient measure to enhance the power system resilience. However, the conventional planning models are generally insufficient to hedge against the extreme weather-related outages mainly due to two reasons. 1) The conventional planning models determine the optimal placement of distribution system assets to meet the new and growing demand, such as construction or upgrade of substations, and installation of distributed generation [5]-[6]. However, these assets have no direct consideration of distribution system resilience from natural disasters. 2) The $\mathrm{N}-1$ criteria is widely implemented in the conventional planning models to ensure the secure operation of distribution systems, known as the reliability constrained planning model (RL-CPM) [7]-[8]. The RL-CPM is efficient for the single fault in distribution systems, but insufficient for the extreme weather-related attacks which can damage multiple components simultaneously. In this case, it is necessary to protect distribution systems from natural disasters by allocating the defensive resources, such as hardening vulnerable distribution lines and allocating backup distributed generators (B-DGs) [9].

Hardening distribution lines by undergrounding or reinforcing materials can make these lines less vulnerable to extreme weather events such as hurricanes, thereby can continuously supply the critical loads. Moreover, when a natural disaster attacks large area and causes multiple line outages, some islands will be created which are isolated from the main grid. In this case, the controllable generators with the self-start ability in these islands can be utilized to restore critical loads [10]-[12]. Therefore, the development of resilience oriented planning model (RS-OPM) for the optimal allocation of defensive resources can effectively alleviate the outage impacts from natural disasters. 
However, the stochastic nature of natural disasters and their nonlinear impacts on system outages impose a considerable challenge on the RS-OPM design. There are generally two types of extreme weather-related uncertainties affecting the planning decisions: the first type of uncertainty is from the offensive resources (ORs) of natural disasters, such as the force uncertainty of hurricanes, due to the inaccurate information to forecast natural disasters in the planning stage. The OR-related uncertainty indicates that distribution systems will suffer from natural disasters with the random intensity. The second type of uncertainty is the nonlinear impacts of a given natural disaster on the branches of distribution systems, such as the number and location of line outages represented as the probability distribution of line outages (PDLO), which are generally unavailable for network planning due to the random fragility of assets and their ambience. These uncertainties should be simultaneously considered in the RS-OPM.

Certain research has been conducted to allocate the defensive resources by considering the weather-related uncertainties with multi-scenario based stochastic method [13]. In [14], the hardening decision-dependent uncertainty of line damage status was considered, and a novel modeling strategy was proposed to generate several representative scenarios. Hence, a twostage stochastic mixed integer problem was formulated to harden existing distribution lines and allocate B-DGs and automatic switches, i.e., the first stage determined the planning decisions and the second stage determined the operational strategies under multiple outage scenarios. In [15], the spatiotemporal correlation among various uncertainties was characterized with a hybrid independent stochastic process, including the random number, position and time of line outage, repair time, as well as the power demand. Then, a multiscenario based planning model was proposed to deploy defensive resources. The mobile energy storages in [16] and mobile emergency generators in [17] were pre-positioned strategically in distribution systems considering multiple outage scenarios, and these facilities will be further dispatched to restore critical loads and reconfigure provisional microgrids.

The multi-scenario based stochastic method is effective to address the OR uncertainty because a given distribution system is assumed to be attacked by limited natural disasters. However, multiple probability-weighted scenarios should be generated under a predefined PDLO, as any inaccurate prediction for the PDLO would lead to a suboptimal or infeasible planning solution. Moreover, it can be a computational challenge to involve all outage scenarios in the optimization model [18]-[19]. For example, if 5 lines can be damaged by the natural disaster in a distribution system with 100 lines, there are scenarios with the number of almost 72 million to be considered, thereby making the computation impractical. Hence, the multi-scenario based stochastic method is unsuitable to model the nonlinear impacts of a given disaster on system outages due to the uncertainty of PDLO.

Since there are inherent limitations concerning the accurate prediction for the PDLO in the multi-scenario based stochastic method, some efforts have made to deploy defensive resources with the robust optimization [20]-[21]. The robust optimization is modeled based on the worst case of weather-related outages in a given uncertainty set, and the uncertainty 
set is generally formulated based on the N-K criteria, where the total number of facilities is $\mathrm{N}$, and the number of damaged facilities is predefined as $\mathrm{K}$ [22]-[25]. For example, in [22] and [23], an N-K based conventional robust model (CRM) was proposed to enhance the planning and operation resilience of distribution systems. Furthermore, to capture the spatial and temporal dynamics of a given natural disaster, the traditional $\mathrm{N}-\mathrm{K}$ criteria was extended to a multi-stage and multi-zone based uncertainty set in [26]. In [27], a predetermined PDLO was integrated in the uncertainty set based on the Claude Shannon's information theory, and the corresponding uncertainty budget in this uncertainty set was pre-assumed as a constant. In [28], the errors of PDLO forecasting were considered based on an ambiguity set, and this ambiguity set contained all possible probability distributions with a distributionally robust model (DRM). In [29], an improved CRM was built for transmission line resilience. In particular, the different values of $\mathrm{K}$ were applied in the $\mathrm{N}-\mathrm{K}$ based uncertainty sets to represent various ORs.

In the CRM, the uncertainty set is developed with no requirement for the accurate PDLO, which shows practicality over the multi-scenario based stochastic method. However, the CRM overestimates the occurrence probability of the worst-case scenario, thereby resulting in an unnecessarily high capital cost, which is termed as conservativeness [30]-[31]. Furthermore, although the DRM in [27] is less conservative than the CRM because all possible PDLOs in the ambiguity set are considered in the DRM, a single ambiguity set is still insufficient to describe the random ORs [29]. Consequently, the CRM and DRM need to be improved to address various types of uncertainties with less conservativeness.

TABle I Comparisons Between the PROPOSEd Model With OTHER EXISTing Models

\begin{tabular}{|c|c|c|c|c|c|c|}
\hline \multirow{2}{*}{ References } & \multicolumn{2}{|c|}{ Targeted systems } & \multirow{2}{*}{$\begin{array}{c}\text { OR } \\
\text { uncertainty }\end{array}$} & \multicolumn{2}{|c|}{$\begin{array}{c}\text { Nonlinear impact of natural disaster on } \\
\text { braches }\end{array}$} & \multirow{2}{*}{ Models } \\
\hline & Transmission & Distribution & & $\begin{array}{c}\text { Line outage uncertainty } \\
\text { (number/location) }\end{array}$ & $\begin{array}{c}\text { PDLO } \\
\text { uncertainty } \\
\end{array}$ & \\
\hline [14]-[17] & & $\overline{\bar{V}}$ & $\overline{\bar{V}}$ & $\sqrt{1}$ & & Stochastic \\
\hline $\begin{array}{l}{[22],[23]} \\
{[26],[27]}\end{array}$ & & $\sqrt{ }$ & & $\sqrt{ }$ & & $\begin{array}{l}\text { Conventional } \\
\text { robust }\end{array}$ \\
\hline$[24],[25]$ & $\sqrt{ }$ & & & $\sqrt{ }$ & & $\begin{array}{l}\text { Conventional } \\
\text { robust }\end{array}$ \\
\hline [28] & & $\sqrt{ }$ & & $\sqrt{ }$ & $\sqrt{ }$ & $\begin{array}{c}\text { Distributionally } \\
\text { robust }\end{array}$ \\
\hline [29] & $\sqrt{ }$ & & $\sqrt{ }$ & $\sqrt{ }$ & & $\begin{array}{l}\text { Improved } \\
\text { robust }\end{array}$ \\
\hline This paper & & $\sqrt{ }$ & $\sqrt{ }$ & $\sqrt{ }$ & $\sqrt{ }$ & \begin{tabular}{|c} 
Improved \\
distributionally \\
robust
\end{tabular} \\
\hline
\end{tabular}

According to the previously reviewed research, there is a research gap to model various weather-related uncertainties with effective methods in the RS-OPM. For this purpose, a multi-disaster-scenario based distributionally robust model (MDS-DRM) is proposed for power lines hardening and B-DGs allocation in distribution systems. In this model, the OR- 
related uncertainty and the random PDLO are simultaneously modelled based on their inherent characteristics. Specifically, the random PDLO is modeled as an ambiguity set based on the moment information, and this set can be developed independently of the outage probability's accuracy. Then, the OR-related uncertainty is described with several probability-weighted scenarios. In these scenarios, different first-order moments are used to describe the random OR with corresponding probabilities. Table 1 compares the proposed model with other existing planning models for resilience improvement. The model comparison demonstrates the novelty of this paper that is the first time to consider random ORs and PDLOs simultaneously in resilient distribution network planning. The key contributions of this paper are summarized as follows:

1) A novel MDS-DRM is proposed for line hardening and DGs allocation in distribution systems, where weather-related uncertainties are addressed by combing the stochastic and distributionally robust model.

2) An effective primal cut based decomposition method is proposed to solve the proposed multi-scenario two-stage distributionally robust model. In this scheme, the MDS-DRM is first reformulated to eliminate the PDLO-related variables, then the reformulation is transformed to the mixed integer linear problem, which can be solved by commercial solvers. Moreover, the proposed decomposition method can converge to the global optimal planning solution.

3) In comparison with the stochastic programming, the proposed model can withstand the forecast errors of the outage probability and improve the computational efficiency and accuracy. In comparison with the robust optimization, the proposed model can withstand various types of uncertainties and overcome the conservativeness of the CRM.

The remaining paper is organized as follows. In Section II, an overview of the improved distributionally robust model is introduced with the model structure. In Section III, the mathematical formulation of the MDS-DRM is developed. In Section IV, the solution method to solve the MDS-DRM is presented. Case studies are performed in Section V. The paper is concluded in Section VI.

\section{Overview of the improved distributionally robust model}

This section provides an overview of the proposed MDS-DRM. First, based on the conceptual resilience curve, it is necessary to integrate the event-related uncertainties and restoration strategies into the network planning to formulate the MDS-DRM. Then, the framework of the proposed MDS-DRM model is provided with clear model structure.

\section{A. Event dependent network planning}

The conceptual resilience curve consisting of 6 operational resilience states is provided in Fig.1 [4]. In the pre-event state, preventive strategies can be adopted to improve the system performance as the curve starting point, such as the network planning where this paper will focus. Then, the OR- and PDLO-related uncertainties exist in the event process and postevent degraded state. Subsequently, operational strategies can be implemented to reconfigure 
the microgrid systems and restore critical loads using the available resources, such as backup DGs. Last, the damaged components will be repaired in the infrastructure repair state, and the system will return to the normal state.

According to the above analysis, the network planning is associated and interdependent with the OR/PDLO uncertainty and the operational strategies. Hence, the OR/PDLO uncertainty and the operational strategies need to be integrated into the planning model to improve the overall system performance during natural disasters.

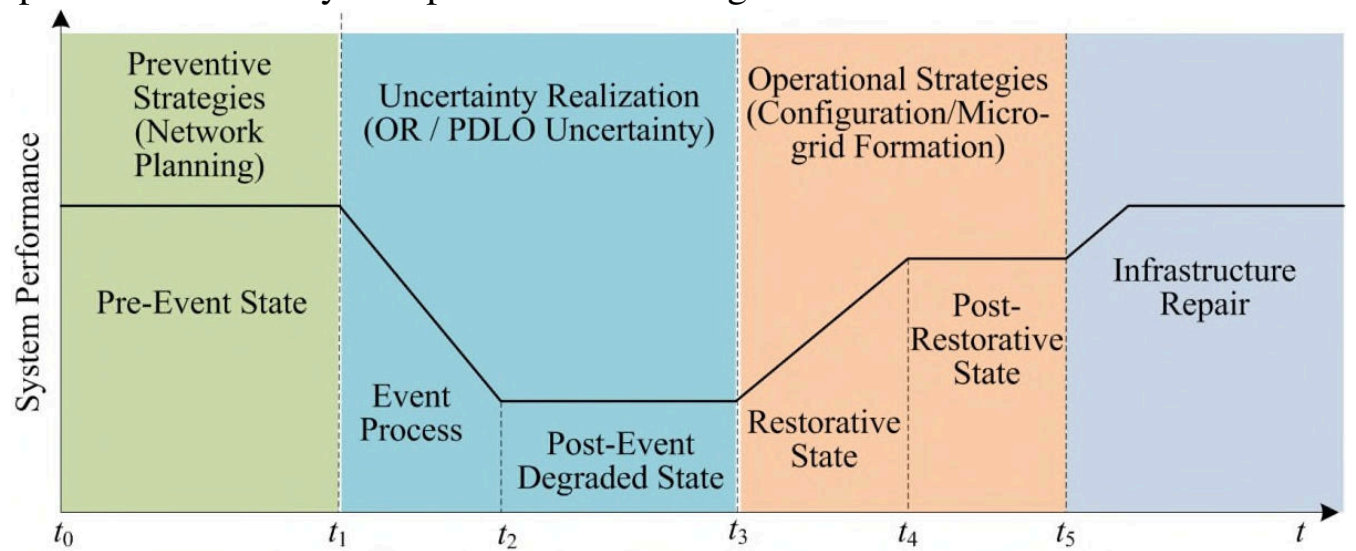

Fig.1 A conceptual resilience curve

\section{B. Framework}

The schematic diagram of the MDS-DRM model is presented in Fig. 2. The objective of the proposed model is to determine the optimal line hardening and B-DG allocation strategies for the resilient network planning. Multi-disaster scenarios are proposed where each scenario has its own ambiguity set to represent different ORs. Moreover, to model the random impacts of a disaster scenario to the line outages, all possible PDLOs incurred by this disaster scenario is considered in the corresponding ambiguity set. Last, in each disaster and line outage scenario, the respective optimal operational strategy is determined by the distribution system operator. It can be seen that the proposed MDS-DRM is a combination of: defender-attackeroperator's interaction, OR- and PDLO-related uncertainties, planning and operational strategies, and stochastic and distributionally robust programming.

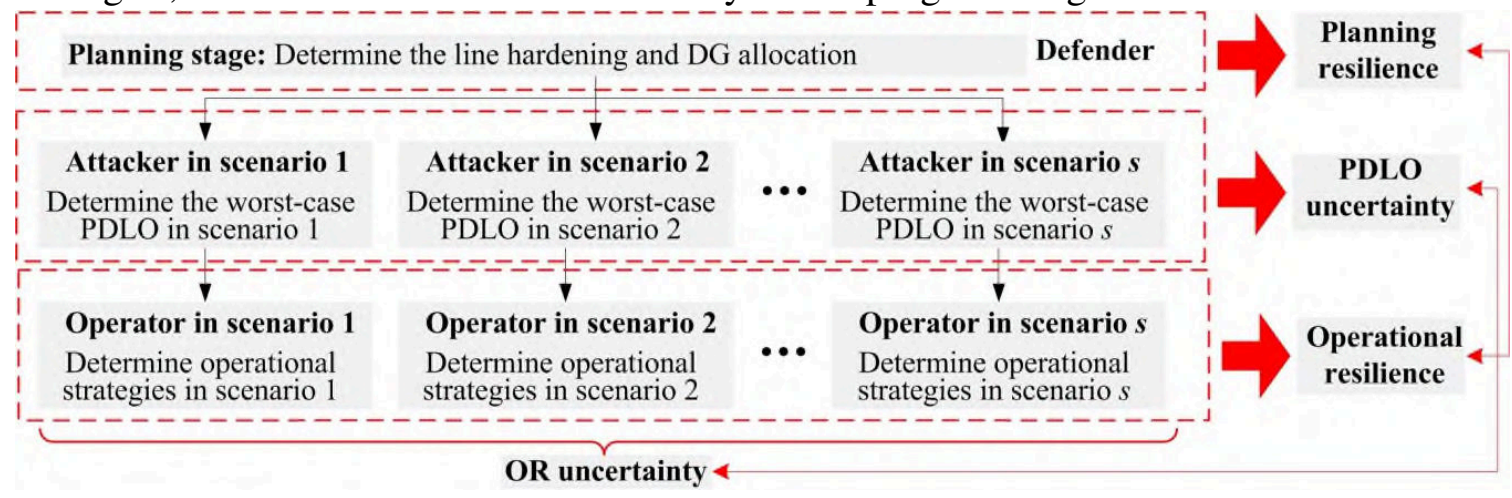

Fig.2 The schematic diagram of the MDS-DRM 


\section{Mathematical formulation of MDS-DRM}

In this section, the mathematical formulation of the proposed MDS-DRM is presented, which consists of the OR and PDLO uncertainty model in Section III-A, the first-stage planning model considering line hardening and DG allocation in Section III-B, and the second-stage operational model considering network reconfiguration and microgrid formation in Section III-C.

\section{A. OR and PDLO uncertainty model (Attacker determination)}

In this section, a novel model combining the stochastic method and DRM is proposed to model the OR and PDLO uncertainties. First, the OR uncertainty is modeled as multiple disaster scenarios based on the stochastic programming. It is noted that certain disasters on a specific distribution system can be obtained from the historical data. Hence, it is reasonable and practical to model the OR uncertainty with the data-driven stochastic method. Then, the nonlinear impacts from a given natural disaster on the distribution line are uncertain, i.e., the random number and location of outages or even the random PDLO. Two moment based ambiguity sets is proposed to model the PDLO uncertainty, which involves the random number and location of outages. It is noted that all PDLOs are involved in the proposed ambiguity sets to achieve the distributional robustness in the planning model.

Specifically, it is assumed that a distribution system will be faced with certain natural disasters, represented by $s_{1}, s_{2}, \cdots, s_{n}$ with the occurrence probability of $\rho^{s 1}, \rho^{s 2}, \cdots, \rho^{s n}$, respectively. Then, with a given disaster denoted as $s$, all PDLOs incurred by the disaster $s$ are included in an ambiguity set $\mathrm{F}^{s}$. Therefore, the scenario set, denoted as $\left\{\mathrm{F}^{s 1}, \mathrm{~F}^{s 2}, \cdots\right.$, $\mathrm{F}^{s n}$ \}, can be formed to model the OR and PDLO uncertainty.

To elaborate the ambiguity set, two samples are presented in this section as shown in (1a) and (1b). Specifically, the ambiguity set $\mathrm{F}^{s}$ in (1a) shows the outage probability of line $i j$ within the defined confidence interval $\left[p_{i j}^{L, s}, p_{i j}^{U, s}\right]$, while the ambiguity set in (1b) constrains the expected number of damaged lines, i.e., $\quad K^{a v e, s}$. It is noted that the first-order moment information, i.e. $\left[p_{i j}^{L, s}, p_{i j}^{U, s}\right]$ and $K^{a v e, s}$, can be obtained from the historical data [30].

$$
\begin{aligned}
& \mathrm{F}^{s}=\left\{\mathrm{P}^{s} \in \mathbf{P}^{s}\left(Z^{s}\right): p_{i j}^{L, s} \leq \mathrm{E}_{\mathrm{P}^{s}}\left[1-z_{i j}^{s}\right] \leq p_{i j}^{U, s}\right\} \\
& \mathrm{F}^{s}=\left\{\mathrm{P}^{s} \in \mathrm{P}^{s}\left(Z^{s}\right): \mathrm{E}_{\mathrm{P}^{s}}\left[\sum_{(i, j) \in \Omega_{B}} z_{i j}^{s}\right] \geq N-K^{a v e, s}\right\}
\end{aligned}
$$

where $\Pi^{s}\left(Z^{s}\right)$ represents the set of all probability distributions on $Z^{s}$, and $Z^{s}$ is built based on the $N-K$ criterion, as shown in (4).

$$
Z^{s}=\left\{\mathbf{z}^{s} \in \mathrm{R}^{\left|\Omega_{B}\right|}: \sum_{(i, j) \in \Omega_{B}}\left[1-\left(1-y_{i j}^{h}\right)\left(1-z_{i j}^{s}\right)\right] \geq N-K^{s}\right\}
$$

Moreover, in various natural disaster scenarios, the corresponding ambiguity sets have different outage lines such as $K^{s}$, different first-order moment information such as confidence intervals $\left[p_{i j}^{L, s}, p_{i j}^{U, s}\right]$, and different expected number of outage lines $K^{a v e, s}$. Such mathematical 
differences indicate the randomness of offensive resources that various natural disasters possess.

Last, the ambiguity set is used to model the uncertain PDLO, and the superiority of this DRM over other probability methods, such as uncertainty sets in stochastic and CRM, can be justified as shown in Table II (taking (1a) as an example), where $p_{i j}^{F I X, s}$ is the fixed PDLO value estimated in stochastic method. Specifically, in stochastic programming, the PDLO is fixed as a constant, and representative scenarios are sampled from the uncertainty set. However, the PDLO forecast errors cannot be well addressed in the stochastic method, and the model only can hedge against finite outage scenarios. In CRM, the PDLO is ignored, and only a specific scenario causing the most serious damage to distribution systems is considered Therefore, the planning decision of CRM is generally conservative and expensive. Comparatively, the moment based ambiguity set of DRM contains all possible PDLOs, which is considered as optimal over the inherent disadvantages of stochastic and robust method in addressing PDLO. Consequently, the proposed ambiguity set is a more feasible method to represent the PDLO uncertainty.

TABLE II COMPARISON OF STOCHASTIC METHOD, CRM AND DRM

\begin{tabular}{cccc}
\hline & Stochastic Method & DRM & CRM \\
\hline \multirow{2}{*}{ Uncertainty Modelling } & Finite scenarios sampling & Ambiguity Set & Uncertainty Set \\
& from $\mathrm{E}_{\mathrm{P}^{s}}\left[1-z_{i j}^{s}\right]=p_{i j}^{\mathrm{FIX}, s}$ & $p_{i j}^{L, s} \leq \mathrm{E}_{\mathrm{P}^{s}}\left[1-z_{i j}^{s}\right] \leq p_{i j}^{U, s}$ & $0 \leq \mathrm{E}_{\mathrm{P}^{s}}\left[1-z_{i j}^{s}\right] \leq 1$ \\
Features & $\begin{array}{c}\text { Suboptimal, finite } \\
\text { robustness }\end{array}$ & Optimal, distributional & Suboptimal, conservative \\
& robustness & robustness \\
\hline
\end{tabular}

\section{B. Distribution network planning model (Defender decision)}

In the planning stage, the objective function minimizes the investment costs of line hardening, B-DG allocation and the expectation of load curtailments under the worst-case PDLO, shown as first, second and third part of (3) respectively. It is noted that the load curtailments are utilized to evaluate the system performance degradation during natural disasters, and this metric has been widely adopted to assess the power distribution system resilience [12]-[17]. The feasibility set $\mathrm{Y}$ defines the limits of the defensive resources, as expressed in (4).

$$
\begin{aligned}
& \min _{\mathbf{y} \in \mathrm{Y}}\left\{\sum_{(i j) \in \Omega_{B}} c_{i j}^{h} y_{i j}^{h}+\sum_{i \in \Omega_{N}} c_{i}^{g} y_{i}^{g}+\sum_{s \in S} \rho^{s} \cdot \sup _{\mathrm{P}^{s} \in \mathrm{F}^{s}} \mathrm{E}_{\mathrm{P}^{s}}\left[Q\left(\mathbf{y}, \mathbf{z}^{s}\right)\right]\right\} \\
& \mathrm{Y}=\left\{\mathbf{y} \mid \sum_{(i j) \in \Omega_{B}} y_{i j}^{h} \leq H, \sum_{i \in \Omega_{N}} y_{i}^{g} \leq G\right\}
\end{aligned}
$$

where $\mathrm{E}_{\mathrm{P}^{s}}[\cdot]$ is the expectation under distribution $\mathrm{P}^{s} ; \mathrm{F}^{s}$ is the ambiguity set for the distribution $\mathrm{P}^{s}$; and $Q\left(\mathbf{y}, \mathbf{z}^{s}\right)$ is defined as the minimum load shedding costs given $\mathbf{y}$ and $\mathbf{z}^{\mathrm{s}}$ in the disaster $s$, which will be expressed in Section III-C.

\section{Network reconfiguration and microgrid formation (Operational strategy)}


Under a given planning decision $\mathbf{y}$ and attacker determination $\mathbf{z}^{s}$ in the natural disaster $s$, the resilience of distribution systems is further enhanced by post-disaster operational strategies of network reconfiguration and microgrid formation. Specifically, the objective function in the operational stage is to minimize the penalty costs for load shedding, as shown in (5a), and this function (5a) has been considered in the planning objective function (3) as load curtailments.

$$
Q\left(\mathbf{y}, \mathbf{z}^{s}\right)=\min _{\mathbf{x}^{s}, \mathbf{v}^{s}} \sum_{t \in T} \sum_{i \in \Omega_{N}} c_{i}^{l} \Delta P_{i t}^{s}
$$

The network reconfiguration and microgrid formation are integrated into the planning model to improve the operational resilience of distribution systems. These constraints are classified into operational constraints and topology constraints [23]. The operational constraints (5b)- $(5 \mathrm{~g})$ are represented by the linearized DistFlow model [32], which is widely adopted in distribution system planning [23], [26], [27].

$$
\begin{aligned}
& \left\{\begin{array}{l}
\sum_{j \in m(i)} P_{i j, t}^{s}-\sum_{j \in n(i)} P_{j i, t}^{s}=P_{i t}^{g, s}-\left(P_{i t}^{L}-\Delta P_{i t}^{s}\right) \\
\sum_{j \in m(i)} Q_{i j, t}^{s}-\sum_{j \in n(i)} Q_{j i, t}^{s}=Q_{i t}^{g, s}-\left(Q_{i t}^{L}-\Delta Q_{i t}^{s}\right)
\end{array} \quad \forall i \in \Omega_{N}\right. \\
& \left\{\begin{array}{l}
U_{i t}^{s}-U_{j t}^{s}-\left(R_{i j} P_{i j, t}^{s}+X_{i j} Q_{i j, t}^{s}\right) / U_{0} \leq \mathrm{M}\left(1-v_{i j}^{q, s}\right) \\
U_{i t}^{s}-U_{j t}^{s}-\left(R_{i j} P_{i j, t}^{s}+X_{i j} Q_{i j, t}^{s}\right) / U_{0} \geq-\mathrm{M}\left(1-v_{i j}^{q, s}\right)
\end{array} \quad \forall(i j) \in \Omega_{B}\right.
\end{aligned}
$$

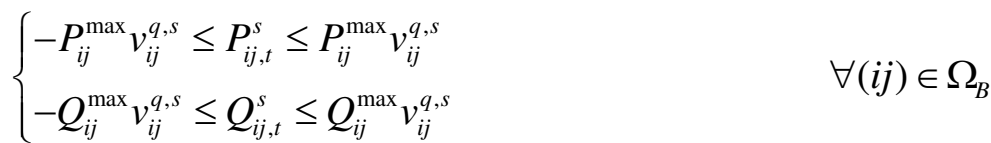

$$
\begin{aligned}
& 0 \leq P_{i t}^{g, s} \leq P_{i}^{\max } y_{i}^{g}, 0 \leq Q_{i t}^{g, s} \leq Q_{i}^{\max } y_{i}^{g} \quad \forall i \in \Omega_{N} \\
& 0 \leq \Delta P_{i t}^{s} \leq P_{i t}^{L}, 0 \leq \Delta Q_{i t}^{s} \leq Q_{i t}^{L} \quad \forall i \in \Omega_{N} \\
& U_{i}^{\min } \leq U_{i t}^{s} \leq U_{i}^{\max } \quad \forall i \in \Omega_{N}
\end{aligned}
$$

where Eqs. (5b) and (5c) are the linearized DistFlow model; Eq. (5d) constrains the line power flow; Eq. (5e) constrains the load curtailment; Eq. (5f) is the DG output constraint; and Eq. (5g) constrains the nodal voltage. Additionally, $v_{i j}^{q . s}$ is the binary variable, with the value of 1 if the final status of line $i j$ is in-service in the scenario $s$, and 0 otherwise. In addition, the final status of line $i j$, i.e., $v_{i j}^{q, s}$, is determined by the line hardening decision $y_{i j}^{h}$, damaged status $z_{i j}^{s}$ and switch status $v_{i j}^{w, s}$, and their relationship can be illustrated in Fig. 3. Specifically, if the line $i j$ is either hardened $\left(y_{i j}^{h}=1\right)$ or not damaged $\left(z_{i j}^{s}=1\right)$ in the scenario $s$, the line $i j$ will be available $\left(v_{i j}^{d, s}=1\right)$ in the operational stage to restore critical loads. The relationship of $v_{i j}^{d, s}, y_{i j}^{h}$ and $z_{i j}^{s}$ is modeled in (5h-1). Moreover, the available line $i j\left(v_{i j}^{d, s}=1\right)$ is further defined as "normal line" $\left(v_{i j}^{q, s}=1\right)$ if the available line $i j$ is switched on $\left(v_{i j}^{w, s}=1\right)$ by the operational strategies. These relationships are formulated as the nonlinear equation (5h-2), and (5h-2) can be linearized as the linear constraints (5h-3). 


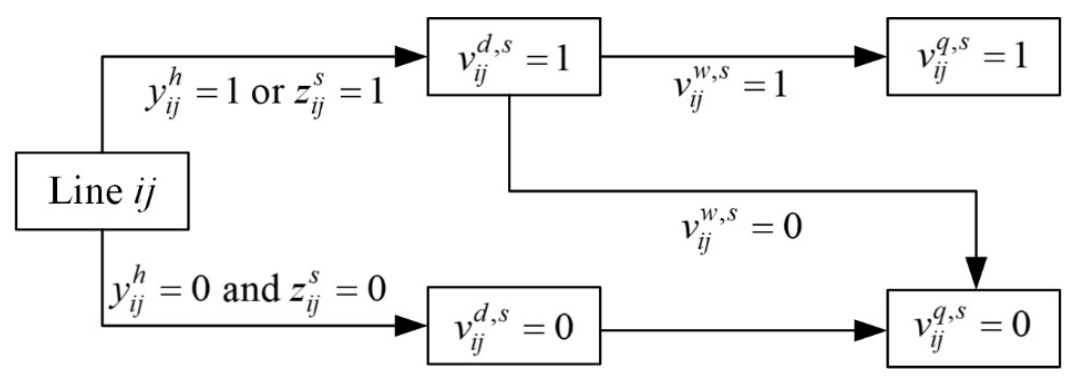

Fig.3 The state of line $i j$ in planning and operation stages

$$
\begin{array}{ll}
v_{i j}^{d, s}=y_{i j}^{h}+z_{i j}^{s}-y_{i j}^{h} \cdot z_{i j}^{s}, & \forall(i j) \in \Omega_{B} \\
v_{i j}^{q, s} \leq v_{i j}^{d, s} \cdot v_{i j}^{w, s}, & \forall(i j) \in \Omega_{B} \\
v_{i j}^{q, s} \leq v_{i j}^{d, s}, v_{i j}^{q, s} \leq v_{i j}^{w, s}, v_{i j}^{q, s} \geq v_{i j}^{d, s}+v_{i j}^{w, s}-1, & \forall(i j) \in \Omega_{B}
\end{array}
$$

where $v_{i j}^{d, s}$ is the binary variable, with the value of 1 if line $i j$ is either hardened or not damaged in the scenario $s$, and 0 otherwise; and $v_{i j}^{w, s}$ is the binary variable, the value is 1 if line $i j$ is switched on in the scenario $s$, and 0 otherwise.

To improve the network resilience, distribution systems are operated radially by introducing the topology constraints. The distribution network is radial if and only if: a) the number of closed branches equals to the number of buses minus the number of islands, and b) the connectivity in islands is guaranteed [33]. To match these conditions, a singlecommodity flow method [12] is adopted here. Specifically, Eq. (5i) and (5j) are formulated to meet conditions a) and b), respectively.

$$
\begin{array}{ll}
\sum_{(i j) \in \Omega_{B}} v_{i j}^{q, s}=N_{b u s}-\sum_{i \in \Omega_{N}} v_{i}^{r, s} & \\
\left\{\begin{array}{lc}
\sum_{j \in m(i)} F_{i j}^{s}-\sum_{j \in n(i)} F_{j i}^{s} \geq-1-\mathrm{M} \cdot v_{i}^{r, s} \cdot\left(y_{i}^{g}+v_{i}^{e, s}\right) & \forall i \in \Omega_{N} \\
\sum_{j \in m(i)} F_{i j}^{s}-\sum_{j \in n(i)} F_{j i}^{s} \leq-1+\mathrm{M} \cdot v_{i}^{r, s} \cdot\left(y_{i}^{g}+v_{i}^{e, s}\right) & \forall i \in \Omega_{N} \\
-\mathrm{M} \cdot v_{i j}^{q, s} \leq F_{i j}^{s} \leq \mathrm{M} \cdot v_{i j}^{q, s} & \forall i j \in \Omega_{B}
\end{array}\right.
\end{array}
$$

In Eq. (5i), each provisional microgrid (i.e., island) must contain one root bus. Hence, the summation of $v_{i}^{r, s}$ is identical to the number of islands. In Eq. (5j), it is assumed that there is the fictitious load of "1" at all non-root-buses. In this case, all non-root-buses should be connected to the root buses to meet the fictitious power balance in (5j). Moreover, the bus $i$ is qualified for the root bus if one of the following conditions is met: 1) a B-DG is located at bus $i$; 2) bus $i$ is at one end of faulted lines. In this paper, $\left(y_{i}^{g}+v_{i}^{e, s}\right)$ is introduced to indicate whether bus $i$ has potential to be chosen as the root bus in the scenario $s$, and it constrains the value of $v_{i}^{r, s}$ as shown in (51) and Fig. 4. Specifically, if $y_{i}^{g}+v_{i}^{e, s}=0$, the value of the binary variable $v_{i}^{r, s}$ is 0 according to (51), and the fictitious load of " 1 " exists at bus $i$ according to (5j). If $y_{i}^{g}+v_{i}^{e, s}=1$, the node $i$ is qualified, and the node $i$ will be further selected as the root bus when $v_{i}^{r, s}=1$. 


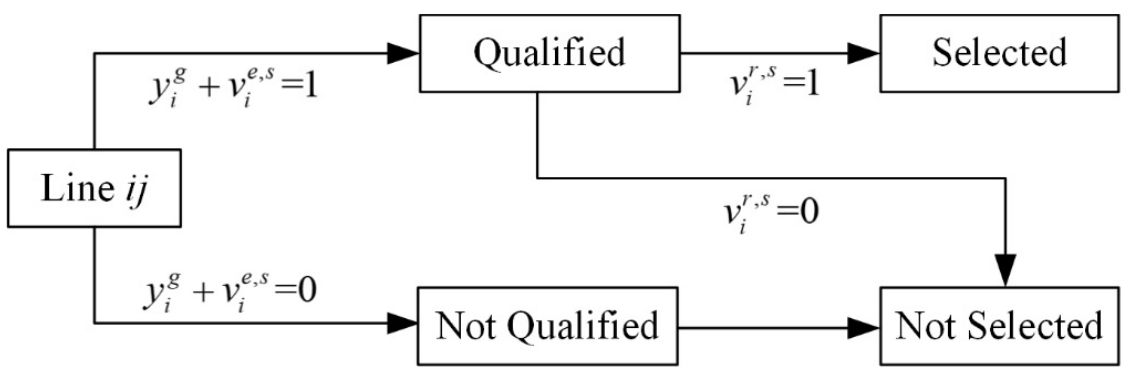

Fig.4 The selection of the qualified root bus

$v_{i}^{r, s} \leq y_{i}^{g}+v_{i}^{e, s} \quad \forall i \in \Omega_{N}$

The value of $v_{i}^{e, s}$ is determined by the availability status of line $i j$. Specifically, if the line $i j$ is unavailable (i.e., $v_{i j}^{d, s}=0$ ), $v_{i}^{e, s}=v_{j i}^{e, s}=1$; Otherwise, $v_{i}^{e, s}=v_{j i}^{e, s}=0$. Therefore, the mathematical formulation of $v_{i}^{e s s}$ can be modeled as shown in (51).

$$
\sum_{j \in \Omega_{N}}\left(1-v_{i j}^{d, s}\right) / \mathrm{M} \leq v_{i}^{e, s} \leq \sum_{j \in \Omega_{N}}\left(1-v_{i j}^{d, s}\right) \quad \forall i \in \Omega_{N}
$$

Next, there are nonlinear relationships in (5j), i.e., $v_{i}^{r, s} v_{i}^{e, s}$ and $v_{i}^{r, s} y_{i}^{g}$. Consequently, binary variables $v_{i}^{c 1, s}$ and $v_{i}^{c, s}$ are introduced to linearize $v_{i}^{r, s} v_{i}^{e, s}$ and $v_{i}^{r, s} y_{i}^{g}$, respectively, as shown in $(5 \mathrm{~m})$. Then, $(5 \mathrm{j})$ can be transformed to a linear form as shown in $(5 \mathrm{n})$.

$$
\begin{gathered}
\begin{cases}v_{i}^{c 1, s} \leq v_{i}^{r, s}, v_{i}^{c 1, s} \leq v_{i}^{e, s}, v_{i}^{c 1, s} \geq v_{i}^{r, s}+v_{i}^{e, s}-1 & \forall i \in \Omega_{N} \\
v_{i}^{c 2, s} \leq v_{i}^{r, s}, v_{i}^{c 2, s} \leq y_{i}^{g}, v_{i}^{c 2, s} \geq v_{i}^{r, s}+y_{i}^{g}-1 & \forall i \in \Omega_{N}\end{cases} \\
\begin{cases}\sum_{j \in m(i)} F_{i j}^{s}-\sum_{j \in n(i)} F_{j i}^{s} \geq-1-\mathrm{M} \cdot v_{i}^{c 1, s}-\mathrm{M} \cdot v_{i}^{c 2, s} & \forall i \in \Omega_{N} \\
\sum_{j \in m(i)} F_{i j}^{s}-\sum_{j \in n(i)} F_{j i}^{s} \leq-1+\mathrm{M} \cdot v_{i}^{c 1, s}+\mathrm{M} \cdot v_{i}^{c 2, s} & \forall i \in \Omega_{N} \\
-\mathrm{M} \cdot v_{i j}^{q, s} \leq F_{i j}^{s} \leq \mathrm{M} \cdot v_{i j}^{q, s} & \forall i j \in \Omega_{B}\end{cases}
\end{gathered}
$$

Consequently, the optimal dispatch model with network reconfiguration and provisional microgrid formation is formulated as a mixed integer linear programming (MILP), as shown in $(50)$.

$\left\{\begin{array}{l}\text { Objective: } \\ \text { Opeartional constraints: }(5 \mathrm{~b})-(5 \mathrm{~g}) \\ \text { Topology constraints: } \quad(5 \mathrm{~h}),(5 \mathrm{i}),(5 \mathrm{k})-(5 \mathrm{n})\end{array}\right.$

In this paper, we mainly focus on the reliable B-DGs with the controllable startup ability, such as gas turbines. The uncontrollable wind power generators are generally insufficient to restore critical customers independently after natural disasters because: 1) the wind power generators are lack of black startup ability; and 2) the wind power quality cannot be guaranteed due to the uncertain and fluctuant output of wind generators. Hence, we focus on the allocation of traditional and practical B-DGs, and the wind generators are not considered as the suitable black start candidates in this paper. 
However, the wind generators in the distribution system can cooperate with other allocated B-DGs to restore critical loads [34], [35]. In the cooperation scheme, the wind generators can be energized by the allocated B-DGs after multiple outages. Then, the B-DGs can serve as the master generator to control the frequency and voltage in the provisional microgrid, and the wind generators can act as the slave generators to provide surplus electricity power [36]. Hence, the operational resilience can be improved by integrating the wind power generator into the load restoration framework.

The proposed MDS-DRM can be extended to consider the wind generators and the windrelated uncertainty by the following steps. First, the wind-related uncertainty can be modeled by the stochastic methods, such as the scenario-based stochastic model that characterizes the correlation of wind-, solar irradiation-, and demand-uncertainty in [7], so that the deterministic model in the operational stage can be reformulated as a stochastic model. Then, the cooperation scheme of the B-DGs and wind power generators in the operational stage can be modeled and integrated into the planning model. Last, the efficient solution method can be designed for the improved MDS-DRM with wind uncertainty. However, this paper focuses on the B-DG allocation and the natural-disaster-related uncertainties. Therefore, the cooperation scheme of B-DG and wind generators for load restoration, as well as the improved MDS-DRM with the wind uncertainty will be investigated as the future work.

\section{Solution algorithm}

The MDS-DRM is a multi-scenario based two-stage distributionally robust model which includes binary variables $\mathbf{y}$ and $\mathbf{v}^{\mathbf{s}}$, continuous variable $\mathbf{x}^{\mathrm{s}}$ and probability distribution variables $\mathrm{P}^{s}$, and these characteristics pose a considerable challenge to solve the model. A novel primal cut based decomposition method is proposed to solve the MDS-DRM with guaranteed convergence and global optimality, and the flowchart is shown in Fig. 5. Specifically, the original MDS-DRM is firstly reformulated to eliminate the probability distributed variables. Then, the two-step proposed decomposition method is employed to solve the equivalent reformulation. First, the reformulation model is decomposed to an upperlevel minimization problem (10) and several lower-level max-min problems with respect to natural disaster scenarios (11). It is noted that the primal cuts are generated from the lowerlevel problems and fed back to the upper-level to refine the planning decision. Subsequently, each lower-level problem is solved individually based on the Column-and-Constraint Generation (CCG) algorithm [37], [38]. 


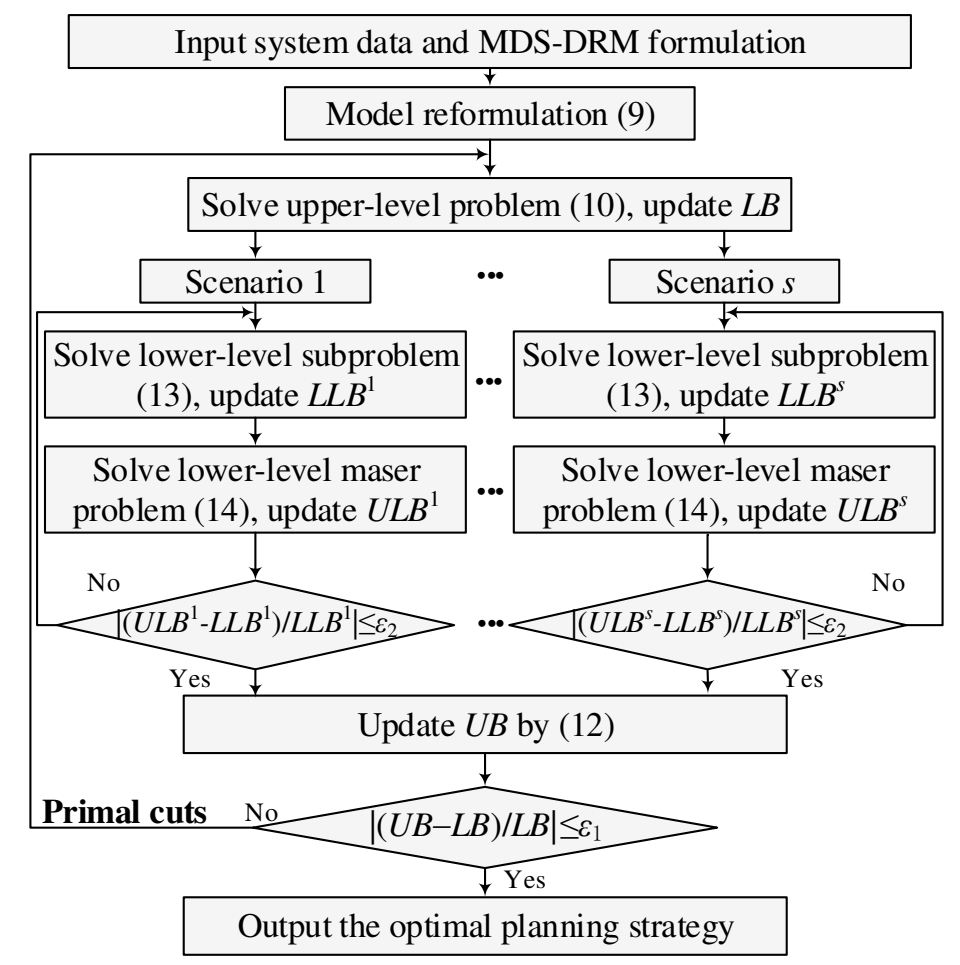

Fig.5 The flowchart of solution method implementation

\section{A. MDS-DRM model reformulation}

The original MDS-DRM is reformulated in (6a)-(6d). Specifically, Eq. (3) and (4) are reduced to (6a) and (6b), and Eq. (1) is reduced to (6c). Moreover, (6d) corresponds to the model (5o).

$$
\begin{array}{ll}
\min & \mathbf{a} \mathbf{y}+\sum_{s \in S} \rho^{s} \cdot \sup _{\mathrm{P}^{s} \in \mathrm{F}^{s}} \mathrm{E}_{\mathrm{P}^{s}}\left[Q\left(\mathbf{y}, \mathbf{z}^{s}\right)\right] \\
\text { s.t. } & \mathbf{A y} \leq \mathbf{b} \\
& \mathrm{F}^{s}=\left\{\mathrm{P}^{s} \in \mathrm{P}^{s}\left(Z^{s}\right): \mathrm{E}_{\mathrm{P}^{s}}\left[\mathbf{B} \mathbf{z}^{s}\right] \leq \mathbf{c}^{s}\right\} \forall s \in S \\
& \begin{cases}Q\left(\mathbf{y}, \mathbf{z}^{s}\right)=\min _{\mathbf{x}^{s}, \mathbf{v}^{s}} \mathbf{d}^{\mathrm{T}} \mathbf{x}^{s} \quad \forall s \in S \\
\mathbf{C y}+\mathbf{D} \mathbf{z}^{s}+\mathbf{E x}^{s}+\mathbf{F} \mathbf{v}^{s} \leq \mathbf{e} \quad \forall s \in S\end{cases}
\end{array}
$$

Next, the worst-case expectation of $\sup \mathrm{E}_{\mathrm{P}^{s}}\left[Q\left(\mathbf{y}, \mathbf{z}^{s}\right)\right]$ is reformulated to an optimization problem, as shown in (7).

$$
\begin{array}{ll}
\sup _{\mathrm{P}^{s} \in \mathrm{F}^{s}} & \mathrm{E}_{\mathrm{P}^{s}}\left[Q\left(\mathbf{y}, \mathbf{z}^{s}\right)\right]=\max _{\mathrm{P}^{s}} \int_{Z^{s}} Q\left(\mathbf{y}, \mathbf{z}^{s}\right) \mathrm{P}^{s}\left(\mathrm{~d} \mathbf{z}^{s}\right) \\
\text { s.t. } & \int_{Z^{s}} \mathbf{B z}^{s} \mathrm{P}^{s}\left(\mathrm{~d} \mathbf{z}^{s}\right) \leq \mathbf{c}^{s} \quad: \quad \boldsymbol{\alpha}^{s} \\
& \int_{Z^{s}} \mathrm{P}^{s}\left(\mathrm{~d} \mathbf{z}^{s}\right)=1 \quad: \quad \beta^{s}
\end{array}
$$


where $\alpha^{s}$ and $\beta^{s}$ are dual variables. In further, (7) is transformed to the dual equation of (8) based on the standard duality, and the derivation procedure is provided in Appendix-A.

$$
\begin{aligned}
& \min _{\boldsymbol{\alpha}^{s} \geq 0, \beta^{s}}\left(\mathbf{c}^{s}\right)^{\cdot} \cdot \boldsymbol{\alpha}^{s}+\beta^{s} \\
& \text { s.t. }\left(\boldsymbol{\alpha}^{s}\right)^{\cdot} \mathbf{B} \mathbf{z}^{s}+\beta^{s} \geq Q\left(\mathbf{y}, \mathbf{z}^{s}\right) \quad \forall \mathbf{z}^{s} \in Z^{s}
\end{aligned}
$$

Then, (8) is integrated into (6), and the equivalent reformulation of (6) is obtained with none probability distribution variables, as shown in (9).

$$
\begin{array}{ll}
\min _{\mathbf{y}, \boldsymbol{a}^{s} \geq 0, \beta^{s}} & \mathbf{a}^{\cdot} \mathbf{y}+\sum_{s \in S} \rho^{s} \cdot\left[\left(\mathbf{c}^{s}\right)^{\bullet} \cdot \boldsymbol{\alpha}^{s}+\beta^{s}\right] \\
\text { s.t. } & \mathbf{A y} \leq \mathbf{b} \\
& \beta^{s} \geq Q\left(\mathbf{y}, \mathbf{z}^{s}\right)-\left(\boldsymbol{\alpha}^{s}\right)^{\bullet} \mathbf{B z}^{s} \quad \forall \mathbf{z}^{s} \in Z^{s}, \forall s \in S \\
& Q\left(\mathbf{y}, \mathbf{z}^{s}\right)=\min _{\mathbf{x}^{s}, \mathbf{v}^{s}} \mathbf{d}^{\mathrm{T}} \mathbf{x}^{s} \quad \forall s \in S \\
& \mathbf{C y}+\mathbf{D} \mathbf{z}^{s}+\mathbf{E} \mathbf{x}^{s}+\mathbf{F} \mathbf{v}^{s} \leq \mathbf{e} \quad \forall s \in S
\end{array}
$$

\section{$B$. The decomposition solution method to solve MDS-DRM}

In this section, the formulation (9), which is a multi-scenario two-stage robust nonlinear optimization model, is solved with a novel primal cut based decomposition method and linear techniques. First, the formulation (9) is decomposed to an upper-level minimization problem (10) and several lower-level max-min problems with respect to different natural disasters (11), as shown in Algorithm 1.

Algorithm 1 The primal cut based decomposition solution method

0 . Initialization. Set the upper bound $U B^{0}=+\infty$, the lower bound $L B^{0}=-\infty$, optimality gap tolerance $\varepsilon_{1}$ and the iteration index $\omega=1$. Define a set of outages in scenario $s$, i.e., $\phi^{s}=\varnothing$.

1. Solve the upper-level problem (10) with given $\phi^{s}$. Record the current optimal planning solution, denoted as $\left(\mathbf{y}^{\omega}, \beta^{s, \omega}, \alpha^{s, \omega}\right)$, and set $L B^{\omega}=O b j U^{\omega}$, where $O b j U^{\omega}$ is the optimal objective value of (10), and the second and third constraints in (10) are the primal cuts from the lower-level problems.

$$
\begin{aligned}
& \min _{\mathbf{y}, \boldsymbol{a}^{s} \geq 0, \beta^{s}} \mathbf{a}^{\cdot} \mathbf{y}+\sum_{s \in S} \rho^{s} \cdot\left[\left(\mathbf{c}^{s}\right)^{\bullet} \cdot \boldsymbol{\alpha}^{s}+\beta^{s}\right] \\
& \text { s.t. } \quad \mathbf{A y} \leq \mathbf{b}
\end{aligned}
$$

$$
\begin{aligned}
& \text { Primal cut 1: } \beta^{s} \geq \mathbf{d}^{\mathrm{T}} \mathbf{x}^{s}-\left(\boldsymbol{\alpha}^{s}\right)^{\bullet} \mathbf{B} \mathbf{z}^{s} \quad \forall \mathbf{z}^{s} \leq \phi^{s} \forall s \in S \\
& \text { Primal cut 2: } \mathbf{C y}+\mathbf{D} \mathbf{z}^{s}+\mathbf{E} \mathbf{x}^{s}+\mathbf{F} \mathbf{v}^{s} \leq \mathbf{e} \quad \forall \mathbf{z}^{s} \leq \phi^{s} \forall s \in S
\end{aligned}
$$

2. Based on the obtained $\left(\mathbf{y}^{\omega}, \beta^{s, \omega}, \alpha^{s, \omega}\right)$, solve the lower-level optimization (11) for each scenario $s$. Record the optimal solution, denoted as $\mathbf{z}^{s, \omega}$, and add $\mathbf{z}^{s, \omega}$ to $\phi^{s}$. Record the optimal value of (11), denoted as $O b j L^{s, \omega}$. 


$$
\begin{aligned}
& \max _{\mathbf{z}^{s} \in Z^{s}}\left\{\min _{\mathbf{x}^{s}, \mathbf{v}^{s}} \mathbf{d}^{\mathrm{T}} \mathbf{x}^{s}-\left(\boldsymbol{\alpha}^{s, \omega}\right)^{\bullet} \mathbf{B} \mathbf{z}^{s}\right\} \\
& \text { s.t. } \quad \mathbf{D} \mathbf{z}^{s}+\mathbf{E} \mathbf{x}^{s}+\mathbf{F} \mathbf{v}^{s} \leq \mathbf{e}-\mathbf{C} \mathbf{y}^{\omega}
\end{aligned}
$$

3. Update $U B^{\omega}$ based on (12).

$$
U B^{\omega}=\min \left\{U B^{\omega-1}, O b j U^{\omega}-\sum_{s \in S} \rho^{s} \cdot \beta^{s, \omega}+\sum_{s \in S} \rho^{s} \cdot O b j L^{s, \omega}\right\}
$$

4. If $\left|\left(U B^{\omega}-L B^{\omega}\right) / L B^{\omega}\right| \leq \varepsilon_{1}$, terminate the process; otherwise, set $\omega=\omega+1$, and go to step 1 .

Convergence: The primal cut based decomposition solution method can be finitely converged to an optimal solution, and the number of iterations is bounded by $Z^{s}$ in (2). The proof for the guaranteed convergence is provided in Section Appendix. C.

In the step 2 of Algorithm 1, the lower-level optimization (11) for each scenario $s$ is a mixed integer two-level max-min optimization programming, and this problem cannot be solved directly by current commercial solvers. In this case, the CCG algorithm is employed to decompose (11) in each scenario into a master problem and a sub-problem. The detailed procedure is shown in Algorithm 2.

\section{Algorithm 2 The CCG Algorithm}

0. Initialization. Set the upper bound of (11) $U L B^{s=+\infty}$, the lower bound of (11) $L L B^{s}=-\infty$, optimality gap tolerance $\varepsilon_{2}$, the iteration index $k=1$, and $\mathbf{z}^{s, k}=\mathbf{1}$. Define a set of binary variables, i.e., $V^{s}=\varnothing$.

1. Fix $\mathbf{z}^{s}$ of (11) as $\mathbf{z}^{s, k}$, and a MILP problem (13) is formed, denoted as the subproblem of (11). Then, solve (13), and add the optimal solution $\mathbf{v}^{s, k}$ into $V^{s}$. Next, record the optimal objective value of (13) as $O b j L L^{s, k}$, and set $L L B^{s}=\max \left\{L L B^{s}, O b j L L^{s, k}\right\}$.

$$
\begin{aligned}
& \min _{\mathbf{x}^{s}, \mathbf{v}^{s}} \mathbf{d}^{\mathrm{T}} \mathbf{x}^{s}-\left(\boldsymbol{\alpha}^{s, \omega}\right)^{\bullet} \mathbf{B} \mathbf{z}^{s, k} \\
& \text { s.t. } \quad \mathbf{E} \mathbf{x}^{s}+\mathbf{F} \mathbf{v}^{s} \leq \mathbf{e}-\mathbf{C y}^{\omega}-\mathbf{D} \mathbf{z}^{s, k}
\end{aligned}
$$

2. Based on the given $V^{s}$, the master problem of (11) is formed as (14). Then, solve (14), and record the optimal attack decision as $\mathbf{z}^{s, k+1}$. In addition, set $U L B^{s}=O b j L U^{s, k}$, where ObjLU $U^{s, k}$ is the objective value of (14).

$$
\begin{array}{ll}
\max _{\mathbf{z}^{s} \in Z^{s}}\left\{\eta^{s}-\left(\boldsymbol{\alpha}^{s, \omega}\right)^{\bullet} \mathbf{B} \mathbf{z}^{s}\right\} & \\
\text { s.t. } & \eta^{s} \leq \min _{\mathbf{x}^{s}} \mathbf{d}^{\mathrm{T}} \mathbf{x}^{s} \quad \forall \mathbf{v}^{s} \in V^{s} \\
& \mathbf{E} \mathbf{x}^{s} \leq \mathbf{e}-\mathbf{C} \mathbf{y}^{\omega}-\mathbf{F} \mathbf{v}^{s}-\mathbf{D} \mathbf{z}^{s} \quad \forall \mathbf{v}^{s} \in V^{s}
\end{array}
$$

3. If $\left|\left(U L B^{s}-L L B^{s}\right) / L L B^{s}\right| \leq \varepsilon_{2}$, terminate the process and set $\mathbf{z}^{s, \omega}=\mathbf{z}^{s, k}$; otherwise, set $k=k+1$, and go to step 1 . 
In Algorithm 2, the sub-problem (13) is a MILP problem which can be solved by commercial solvers such as CPLEX. However, the master problem (14) is a max-min problem which cannot be solved directly. Hence, (14) is further transformed to a single level problem (15) based on the strong duality theory.

$$
\begin{array}{lr}
\max _{\mathbf{z}^{s} \in Z^{s}}\left\{\eta^{s}-\left(\boldsymbol{\alpha}^{s, \omega}\right)^{\bullet} \mathbf{B} \mathbf{z}^{s}\right\} & \\
\text { s.t. } \eta^{s} \leq\left(\mathbf{e}-\mathbf{C y}^{\omega}-\mathbf{F} \mathbf{v}^{s}-\mathbf{D} \mathbf{z}^{s}\right)^{\cdot} \cdot \lambda^{s} & \forall \mathbf{v}^{s} \leq V^{s} \\
\mathbf{E}^{\mathrm{T}} \cdot \boldsymbol{\lambda}^{s} \leq \mathbf{d} & \forall \mathbf{v}^{s} \leq V^{s} \\
\lambda^{s} \leq \mathbf{0} & \forall \mathbf{v}^{s} \leq V^{s}
\end{array}
$$

where $\lambda^{s}$ is the dual variable of (14c). Then, to transform (15) to an MILP problem, the bilinear term $\left(\mathbf{D} z^{s}\right)^{\square} \cdot \lambda^{s}$ of (15c) is linearized with a big "M" method, as shown in (16).

$$
\left\{\begin{array}{l}
-\mathrm{M} \cdot z_{j}^{s} \leq \mu_{i j}^{s} \leq \mathrm{M} \cdot z_{j}^{s} \\
\lambda_{i}^{s} D_{i j}-\mathrm{M} \cdot\left(1-z_{j}^{s}\right) \leq \mu_{i j}^{s} \leq \lambda_{i}^{s} D_{i j}+\mathrm{M} \cdot\left(1-z_{j}^{s}\right)
\end{array}\right.
$$

where $\mu_{i j}^{s}$ is a supplementary variable which equals to $\lambda_{i}^{s} \cdot D_{i j} \cdot z_{j}^{s}$, and $\lambda_{i}^{s}, D_{i j}$ and $z_{j}^{s}$ are elements in $\lambda^{s}, \mathbf{D}$ and $\mathbf{z}^{s}$, respectively. Consequently, (14) is transformed to an MILP problem, and the MDS-DRM is finally decomposed to several MILPs.

It is noted that the decomposition method and the CCG method dynamically update the lower and upper boundaries of (9) and (11), respectively. Therefore, these algorithms can converge to a global optimal solution [37], [38]. In addition, the model simplification and equivalent reformulation are capable of addressing complex nonlinear problems with no compensation on accuracy. Consequently, a global optimal planning strategy can be obtained by applying the proposed solution methods.

The primal cut based solution method decomposes the MDS-DRM into several MILP problems. Hence, the computational efficiency of the solution method mainly depends on the size and complexity of these MILPs. In general, MILP problems might be NP-hard due to the inevitable binary variables. However, there are several advanced commercial solvers and effective solution methods to solve the large-scale MILP problems, such as the Branch \& Bound algorithm embedded in the CPLEX and Gurobi. In addition, the size of some MILPs, such as (15) of the CCG algorithm, can grow with the iteration number and increase the computational burden. However, the iteration number of the CCG method has been proven to be limited and acceptable in several studies [22], [23], [38], and the effectiveness of the CCG method will be further demonstrated in the numerical tests of this paper. Moreover, some techniques can be utilized to reduce the computational burden. First, not all branches are critical to be hardened, and only certain nodes can serve as the candidates for the B-DG allocation. Hence, non-critical lines or non-allocable nodes can be pre-excluded by the risk analysis method [39], and the suitable candidate nodes for the B-DG allocation can be chosen according to the site, access and facility requirements. By doing this, the number of binary variable can be reduced to improve the computational efficiency. Then, some computing 
methods can be utilized, such as the cloud computing method, which can solve the largescale MILPs within seconds.

The proposed primal cut based solution method is more efficient than the candidate solution methods in the existing literature, such as the progressive hedging $(\mathrm{PH})$ algorithm for the multi-scenario based stochastic method [14], and the CCG method for the robust optimization [34]. The $\mathrm{PH}$ methods aims to decompose the stochastic model into multiple sub-problems to reduce the computational burden. However, the PH algorithm is based on the assumption that the number and location of damaged lines are pre-determined in all scenarios. This assumption is challenged by the MDS-DRM because only the PDLO information is utilized. The CCG method is effective for the two-stage robust optimization. However, the CCG method is only efficient for the uncertainty set concerning a single natural disaster, but not applicable to the ambiguity sets with multiple independent disasters.

\section{Case studies}

In this section, case studies are performed in the modified 13-node, 33-node and 135-node distribution systems to validate the effectiveness of the proposed MDS-DRM. All simulations are implemented using GAMS 23.7/CPLEX 12.3 platform on a computer with a core $\mathrm{i} 5,3.2 \mathrm{GHz}$ processor and $4 \mathrm{~GB}$ RAM.

\section{A. Case study in IEEE 13-node distribution system}

In the modified IEEE 13-node distribution system, lines 6,10 and 13 are added [22]. The active power demand at each node is shown in Fig.6 with total load of $1155.35 \mathrm{~kW}$ (shown as italics numbers in the figure). DGs with $100 \mathrm{~kW}$ capacities are served as candidates to be allocated. It is assumed the capital cost of DGs is $\$ 1500 / \mathrm{kW}$ [9], hardening cost for each line is $\$ 400$ [23], and will be valid for 10 years. The penalty cost for load shedding is $\$ 14 / \mathrm{kWh}$ [9].A natural disaster is considered with the duration of $24 \mathrm{~h}$, so that the simulation horizon is set as $24 \mathrm{~h}$ in this paper.

The case study in 13-node distribution system is organized as follows. First, the implementation of MDS-DRM with simulation results are provided. The combination method of the stochastic model and DRM is explained. Then, the superiority of the joint consideration of random OR and PDLO is verified by comparative experiments in the second section. Subsequently, the sensitivity of planning decisions to the OR and PDLO is studied in the third section. Last, the effectiveness of the network planning and operational strategy to enhance the system resilience is demonstrated in the fourth section.

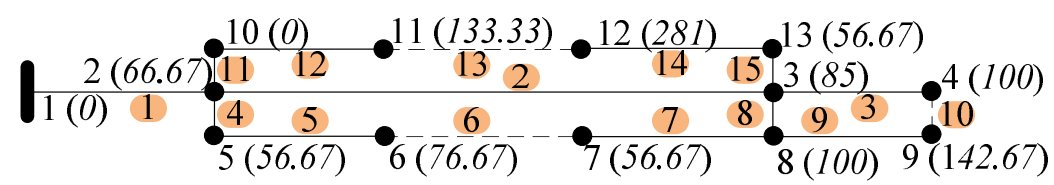

1 Node indices 0 Active load 1 Line indices

Fig.6 The modified IEEE 13-node distribution system 


\section{1) Implementation of the MDS-DRM}

First, the proposed model is implemented in the IEEE 13-node distribution system. We set Case 1: $H=3, G=1$, and the ambiguity set ( $3 b$ ) is employed. In addition, two natural disasters are considered where $K^{s 1}=2, K^{\text {ave,s1 }}=1, \rho^{s 1}=0.7$; and $K^{s 2}=5, K^{\text {ave, } s 2}=2.5, \rho^{s 2}=0.3$; denoted as $\mathbf{s 1}=(2,1,0.7)$ and $\mathbf{s} 2=(5,2.5,0.3)$. The difference between $\mathbf{s 1}$ and $\mathbf{s} 2$ indicates the different offensive resources and occurrence probability of two natural disasters. By applying the proposed model and solution method, the simulation results, as shown in Table III, can be obtained after 200 seconds, which is a practical time for the network planning. With the obtained planning measures, the load curtailments expectation is $243.19 \mathrm{~kW}$, which is the probability-weighted sum of the expected load curtailments in each scenario, i.e., $157.17 \times 0.7+443.90 \times 0.3$. Therefore, $78.95 \%$ of load can be restored by employing the proposed method. Furthermore, in s1, two sets of line outages are detected, and the expected number of line contingencies $K^{\text {ave,s1 }}=1$ is met, i.e. $2 \times 0.5+0 \times 0.5$. Moreover, the expected load curtailments under the worst probability distribution of outages are $157.17 \mathrm{~kW}$ and 443.90 $\mathrm{kW}$ for each disaster denoted as s1 and s2. Consequently, the proposed model considers all possible PDLOs and is able to withstand the worst PDLO in each disaster scenario.

TABLE III SimUlation RESUlTS OF CASE 1

\begin{tabular}{lcc}
\hline \hline Load curtailments expectation $(\mathrm{kW})$ & \multicolumn{2}{c}{243.19} \\
Hardening lines & \multicolumn{2}{c}{$\begin{array}{c}\text { Lines } 1,2 \text { and } 15 \\
\text { Node } 12\end{array}$} \\
DGs placement & $\mathbf{s 1 = ( 2 , 1 , 0 . 7 )}$ & $\mathbf{s 2 = ( 5 , 2 . 5 , 0 . 3 )}$ \\
\hline Natural disaster scenarios & $(11,14), 0.5$ & (None), 0.167 \\
The sets of the worst line outages and corresponding probability & $($ None $), 0.5$ & $(3,4,8), 0.833$ \\
\cline { 2 - 3 } in each $s$ & 157.17 & 443.90 \\
Expected load curtailments in each $\boldsymbol{s}(\mathrm{kW})$ &
\end{tabular}

Then, the implementation of MDS-DRM with ambiguity set (3a) is demonstrated, denoted as $\boldsymbol{C a s e}$ 2. Specifically, according to simulation results of $\boldsymbol{C a s e} \mathbf{1}$, five critical lines, i.e., lines $3,4,8,11$ and 14, are selected, and their outage probability is limited in the confidence interval $[0,0.1]$ in scenario s3, and interval $[0,0.2]$ in scenario s4, respectively. Moreover, the following parameters are set for $\mathbf{s 3}$ and $\mathbf{s} 4: K^{s 3}=2, \rho^{s 3}=0.7, K^{s 4}=5$ and $\rho^{s 4}=0.3$, denoted as $\mathbf{s} 3=(2,[0,0.1], 0.7)$ and $\mathbf{s} 4=(5,[0,0.2], 0.3)$. Under these pre-defined conditions, the simulation results are shown in Table IV. The load curtailments expectation is $252.26 \mathrm{~kW}$ which is probability-weighted sum of the expected load curtailments in each scenario, i.e. $184.57 \times 0.7+410.21 \times 0.3$. The contingency probabilities of critical lines $(3,4,8,11,14)$ are $(0.1,0.1,0,0,0.1)$ in $\mathbf{s} 3$ and $(0.2,0.2,0.2,0.134,0.2)$ in $\mathbf{s 4}$, respectively, and these findings correspond to the preset confidence intervals. Consequently, the proposed approach is effective to improve the system resistance by incorporating the ambiguity set (3a).

TABLE IV Simulation Results of CASE 2

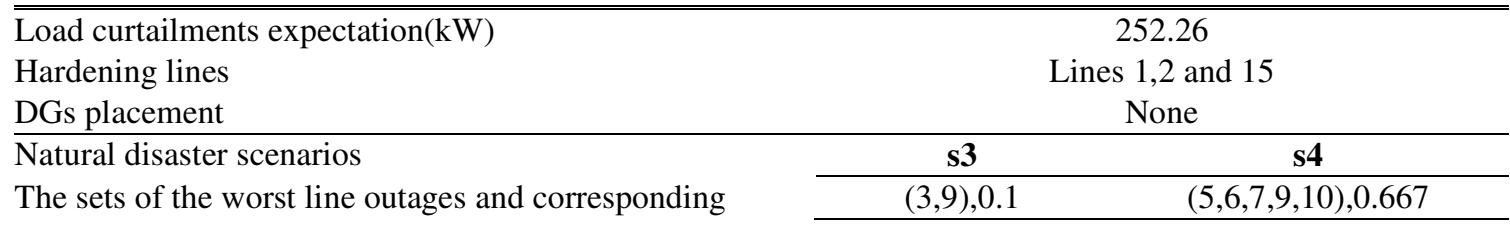


probability in each $s$

$(4,7), 0.1 \quad(4,8,10,12,14), 0.133$

$(9,10), 0.7$

$(3,5,8,11,13), 0.067$

$(12,14), 0.1$

$(3,6,9,11,14), 0.067$

$(3,4,7,9,12), 0.067$

Expected load curtailments in each $s(\mathrm{~kW})$

184.57

410.21

\section{2) Superiority over existing methods}

In this section, to validate the advantages of the joint consideration of the random OR and PDLO, the simulations are conducted to compare the proposed method with existing methods. First, the proposed MDS-DRM is compared with the method only considering the random PDLO, i.e., the DRM, to clarify the necessity to consider the OR uncertainty with the stochastic programming. For this purpose, Case 3 including two simulations with the deterministic realization of OR uncertainty is designed as follows: 1) DRM-1, only the scenario $\mathbf{s 1}$ with the maximum occurrence probability is considered; and 2) DRM-2, the most serious scenario s2 is considered. The simulation results are compared in Table V. Specifically, the first and second columns are planning decisions in Case $\mathbf{1}$ and Case 3. The $3^{\text {rd }} \sim 5^{\text {th }}$ columns are the load curtailments when applying the corresponding planning decisions. The last column is the expected cost totaling the planning cost and penalty for load curtailments under the corresponding planning decisions. It can be seen that when the uncertainty related to the OR is ignored for different type of disasters in Case 3, DRM-1 is only robust and resilient against disaster $\mathbf{s 1}(121.34<157.17$ in $\mathbf{s 1}$, but $907.35>443.9$ in $\mathbf{s 2})$, thereby resulting in more expected load curtailments of $113.95 \mathrm{~kW}$ and higher expected costs of $\$ 23.29 \times 10^{3}$ than the MDS-DRM. Similar analysis can be conducted for the comparison between the MDS-DRM and DRM-2 in Table V. Consequently, the involvement of OR uncertainty into the planning strategies can effectively hedge against various natural disasters with different ORs.

TABle V Comparation Results Between CASE 1 and CASE 3

\begin{tabular}{ccccccc}
\hline \hline & \multirow{2}{*}{ Hardening lines } & \multirow{2}{*}{ DGs placement } & \multicolumn{2}{c}{ Load curtailments $(\mathrm{kW})$} & \multicolumn{2}{c}{ Expected } \\
\cline { 4 - 5 } & & $\mathbf{s 1}$ & $\mathbf{s 2}$ & Expected & cost $\left(10^{3} \$\right)$ \\
\hline MDS-DRM & Lines 1,2,15 & Node 12 & 157.17 & 443.90 & 243.19 & 96.83 \\
DRM-1 & Lines 1,14,15 & None & 121.34 & 907.35 & 357.14 & 120.12 \\
DRM-2 & Lines 1,2,8 & Node 11 & 185.50 & 431.67 & 259.35 & 102.16 \\
\hline \hline
\end{tabular}

In addition to clarify the necessity to consider the OR uncertainty with the stochastic programming, we also quantitatively assess the stochastic solution by some well-known indicators. First, we provide the models to calculate the value of expected value solution $(\mathrm{EV})$, recourse problem solution (RP), expected result of EV (EEV) in the Appendix-B section. Then, the performance indicators, i.e., EEV-EV and EEV-RP in this paper, are evaluated and shown in Table VI. The value of EEV can be utilized to assess the solution quality of the deterministic model. A large value of EEV-EV $\left(\$ 60.88 \times 10^{3}\right)$ means the poor quality of the deterministic model, thereby highlight the necessity to model the OR uncertainty with the stochastic method. EEV-RP measures the expected increase in value from solving the stochastic version of a model rather than the simpler deterministic one. A 
large value of EEV-RP $\left(\$ 23.28 \times 10^{3}\right)$ indicates that it is a valuable work to solve the stochastic model instead of the simpler deterministic one. It is noted that the wait-to-see solution (WS) is also used to assess the stochastic solution in some literatures. However, it is meaningless to our model because: 1) the planning decisions must be applied previously to the occurrence of natural disasters; and 2) the ORs are inevitably varied for a distribution system, so that there is no sense to evaluate the perfect information of the OR. Consequently, the WS and the WS-related indicator, such as the value of perfect information RP-WS, are not adopted to assess the stochastic solution in this paper.

TABLE VI THE VALUE OF PERFORMANCE INDICATORS

\begin{tabular}{|c|c|c|c|c|c|}
\hline Indicator & EV & EEV & $\mathrm{RP}$ & EEV-EV & EEV-RP \\
\hline Value $\left(10^{3} \$\right)$ & 59.23 & 120.11 & 96.83 & 60.88 & 23.28 \\
\hline \multicolumn{6}{|c|}{ TABLE VII SIMULATION RESUlTS OF $\boldsymbol{C A S E} 4$} \\
\hline \multirow{3}{*}{\multicolumn{2}{|c|}{$\begin{array}{l}\text { Hardening lines } \\
\text { DGs placement }\end{array}$}} & DRM-3 & \multicolumn{2}{|c|}{ DRM-4 } & $\overline{\mathrm{CRM}}$ \\
\hline & & Line $1,2,15$ & \multicolumn{2}{|c|}{ Line $1,2,15$} & Line $1,2,8$ \\
\hline & & none & & & Node 11 \\
\hline \multirow{2}{*}{\multicolumn{2}{|c|}{ The worst scenarios }} & (none), 0.25 & $(4,8,1($ & $, 12,14), 0.2$ & \multirow{2}{*}{$(5,12,13), 1$} \\
\hline & & $(12,14), 0.75$ & $(3,9,1$ & $, 10,12), 0.4$ & \\
\hline \multirow{3}{*}{$\begin{array}{l}\text { Expected cost } \\
\qquad\left(\times 10^{3} \$\right)\end{array}$} & s5 & 104.53 & & & 123.73 \\
\hline & s6 & 92.52 & & & 154.90 \\
\hline & $\mathrm{K}=3$ & 179.22 & & & 139.78 \\
\hline
\end{tabular}

In further, the necessity to consider the random PDLOs with DRM is validated by performing the comparative simulations on Case 4 as follows: (1) DRM-3: only the scenario $\mathbf{s 5}=(3,1.5,1)$ is considered, i.e., $K^{s 5}=3, K^{\text {ave }, 55}=1.5, \rho^{s 5}=1$; (2) DRM-4: only the scenario s6 $=(3,[0,0.2], 1)$ is considered, i.e., $K^{s 5}=3, p_{i j}^{L, s}=0, p_{i j}^{U, s}=0.2, \rho^{s 5}=1$; and (3) CRM: the N-K criterion is considered where $K=3$. Then, the simulation results are shown in Table VII. Compared with the DRM, the CRM optimizes the planning measures only based on the worst-case outage scenario, i.e. damaged lines 5, 12 and 13. However, this scenario has low occurrence probability, so the decision from the CRM is too conservative which incurs higher expected cost to withstand the worst-case scenario. To conclude, the employment of the moment-based ambiguity sets makes the planning decisions less conservative than the traditional CRM method.

\section{3) Sensitivity analysis to the $O R$ and PDLO}

In this section, the sensitivity of planning decisions to the OR and PDLO is studied. First, taking the ambiguity set (3a) as an example, Case $\mathbf{5}$ is designed to study the sensitivity to the OR from the aspect of the occurrence probability. Case $\mathbf{5}$ is shown as follows: the probabilities of $(\mathbf{s} 1, \mathbf{s} 2)$ vary from $\{0,1\}$ to $\{1,0\}$, and other conditions are same with Case 1. The expected costs under different probabilities are shown in Fig. 7. It can be seen that the expected cost is reduced with the decrease of the probability of $\mathbf{s} \mathbf{2}$ due to its more detrimental impact on the system. Moreover, it is shown in both Fig.7 and Table VIII that the planning decisions of hardening lines and DGs placement are sensitive to the disaster probability in 
some cases, such as the reduction of s2 probability from 0.8 to 0.6 changes the planning decisions. Therefore, it is necessary to estimate the likelihood of natural disasters precisely in these cases, and prepare two planning strategies according to the probabilities of natural disasters. In contrast, in other cases, e.g., $\{0.6,0.4\}$, the planning decision is identical to $\{0.4$, $0.6)$ and $\{0.8,0.2\}$. In these cases, the planning decision can hedge against the forecast error on disaster probabilities. Furthermore, the MDS-DRM degrades to DRM when the probability is $\{0,1\}$ and $\{1,0\}$ which shows the relationship between the MDS-DRM and the DRM.

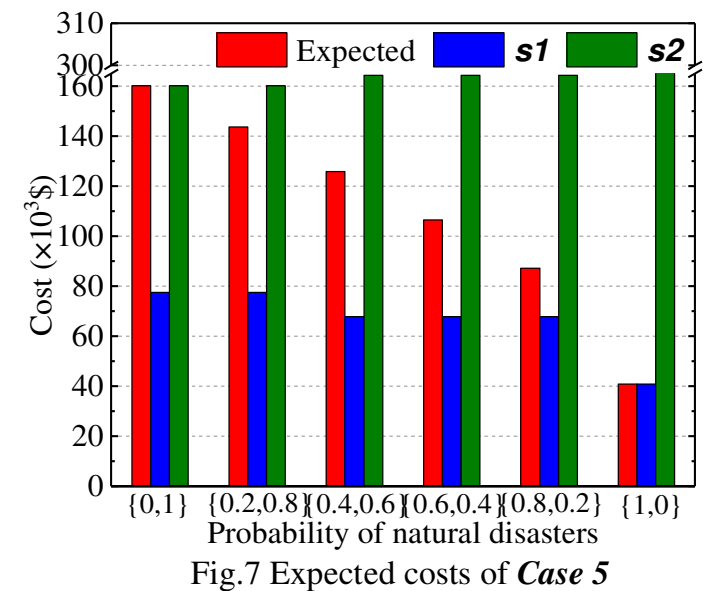

TABle ViII PlanNing DeCisions of CASE 5

\begin{tabular}{ccccccc}
\hline \hline & $\{0,1\}$ & $\{0.2,0.8\}$ & $\{0.4,0.6\}$ & $\{0.6,0.4\}$ & $\{0.8,0.2\}$ & $\{1,0\}$ \\
\hline Hardening lines & $1,2,8$ & $1,2,8$ & $1,2,15$ & $1,2,15$ & $1,2,15$ & $1,14,15$ \\
DGs placement & 11 & 11 & 12 & 12 & 12 & none \\
\hline \hline
\end{tabular}

Case 6 shows the sensitivity analysis to the different PDLOs as follows. Case 6: the value of $K^{\text {ave,s1 }}$ varies from 0.4 to 2 with the increment of 0.4 , and the value of $K^{\text {ave,s2 }}$ varies from 1 to 5 with the increment of 1, with other conditions the same as Case 1. The simulation results are shown in Fig.8. It can be seen that the expected costs become higher with the increase of $K^{a v e, s 1}$ and $K^{\text {ave,s2 }}$. Besides, with the given expected costs, e.g., $\$ 100 \times 10^{3}$ as shown in Fig. 8, this sensitivity analysis can be utilized to search for the upper limit of ORs that the distribution system can withstand. In addition, when $K^{\text {ave,s1 }}=K^{s 1}$ and $K^{\text {ave,s2 }}=K^{s 2}$, the ambiguity set degrades to the N-K criteria, and thus the DRM degrades to the CRM.

\section{4) Effectiveness of planning and operational measures}

In this section, the effectiveness of planning and operational measures is demonstrated. First, we carry out the simulation on Case 7 to validate the efficiency of planning measures in hedging against natural disasters as shown in Fig. 9. Case 7: $(H, G)$ varies from $\{0,0\}$ to $\{5,3\}$ with other conditions identical to Case 1 . It can be seen that the expected costs are significantly reduced with the increase of planning budgets. Moreover, by fixing the budget for the number of DGs placement, e.g., $G=0$, the expected costs are also reduced by the 
increase of the line hardening budgets $H$. The marginal influence of expected cost is relatively steep when $H$ is approaching 0 , because the security of some lines such as lines 1 and 2 is critical for load supplying that have priority to be hardened. Besides, the effectiveness of DGs placement is also critical, especially when the distribution system is damaged seriously, e.g., $H=0$. Finally, with given expected costs, e.g., $100 \times 10^{3} \$$ in Fig.9, the lower bounds of $H$ and $G$ can be identified, which implies the minimum resources that the defender should prepare for natural disasters by employing both line hardening and DG allocation

The effectiveness of operational measures is verified by conducting the simulations on Case 8. Case 8: $G=2$ and the value of $H$ varies from 0 to 5 with and without applying the operational measures, respectively, and other conditions are identical to Case 1. The simulation results are shown in Fig.10. In this case, significant economic benefits of more than $159.81 \times 10^{3} \$$ can be achieved by applying the operational measures when $\mathrm{H}=1$, because the distribution system operator can restore critical loads by network reconfiguration and microgrids formation after a natural disaster based on the proposed strategies in equations (5o). Consequently, combining the planning and operational measures can efficiently improve the resilience of distribution systems.

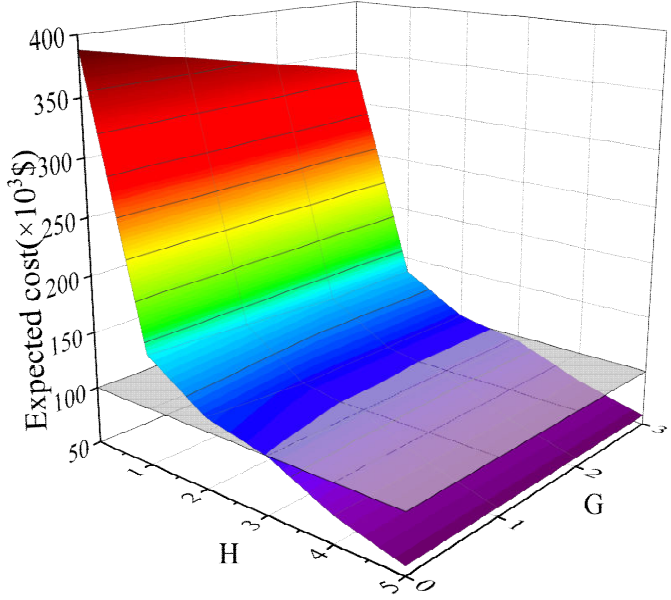

Fig.9 Simulation results of Case 7

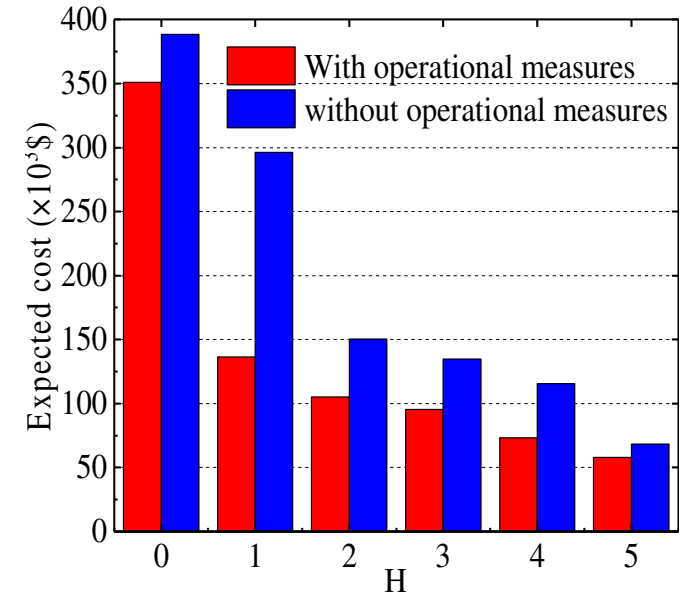

Fig.10 Simulation results of Case 8

\section{B. Case studies in 33-node and 135-node distribution system}

In this section, the effectiveness of the proposed MDS-DRM to larger scaled systems is demonstrated. Then, the precision of the linearized DistFlow model is validated by the comparative simulations with the AC model in load shedding, power loss and voltage amplitude. Last, the effectiveness of the proposed solution algorithm is verified.

1) Effectiveness to 13-node and 135-node systems

The adaptation of the proposed method to larger scaled systems is demonstrated based on the 33-node [40] and 135-node [41] distribution system. First, taking the ambiguity set (3b) as an example, Case $\mathbf{9}$ and Case 10 are studied for the 33-node and 135-node system, respectively. In Case 9, two natural disasters are considered based on the MDS-DRM, i.e., $\mathbf{s} 1=(2,1,0.7), \mathbf{s} 2=(5,2.5,0.3)$. In addition, the budgets for planning resources are set as $H=3$ and $G=2$, and DGs with $400 \mathrm{~kW}$ capacities serve as candidates to be allocated. Similarly, in 
Case 10, DGs with $200 \mathrm{~kW}$ capacities serve as candidates to be allocated, $H=30$ and $G=5$. Moreover, two natural disasters are considered based on the MDS-DRM, i.e., $\mathbf{s 3}=(6,3,0.7)$, $\mathbf{s 4}=(8,4,0.3)$. The computation time of the MDS-DRM implemented in the 33-node and 135 -node system is 870 seconds and 2.5 hours, respectively, and the computation time is feasible for the distribution network planning. The planning and operational strategies are shown in Fig. 11 and Fig. 12, and the load curtailments and costs are listed in Table IX.

\begin{tabular}{|c|c|c|c|c|c|}
\hline & \multicolumn{3}{|c|}{ L Load curtailments (kW) } & \multirow{2}{*}{ Capital cost $\left(\times 10^{3} \$\right)$} & \multirow{2}{*}{ Expected cost $\left(\times 10^{3} \$\right)$} \\
\hline & s1(s3) & s2(s4) & Expected & & \\
\hline Case 9 & 255.00 & 2604.17 & 959.75 & 60.12 & 382.60 \\
\hline Case 10 & 1459.51 & 1901.67 & 1592.157 & 151.20 & 686.16 \\
\hline
\end{tabular}

Then, the effectiveness of the network planning in hedging against natural disasters can be further validated in the post-event operational stage by network configuration and provisional microgrid formation. Specifically, the critical lines being hardened provide the guarantee for the electricity supply to customers, such as the line 1-2, 2-3 in Fig.11, and the main branches in Fig.12. Moreover, the backup DGs can be utilized to energy the provisional microgrids, which are isolated from the main grid due to the line outages. For example, 6 provisional microgrids are formed due to the damaged lines 10-11, 23-24, 48-52, 63-64, 78-79 and 103105 , and these microgrids lose the support from the main grid. However, 5 microgrids, i.e., MG1, MG2, MG3, MG4 and MG5, are still energized by the allocated backup DGs, thereby reducing the load curtailments. Consequently, the combination of planning and operational strategies can effectively improve the distribution system against natural disasters.

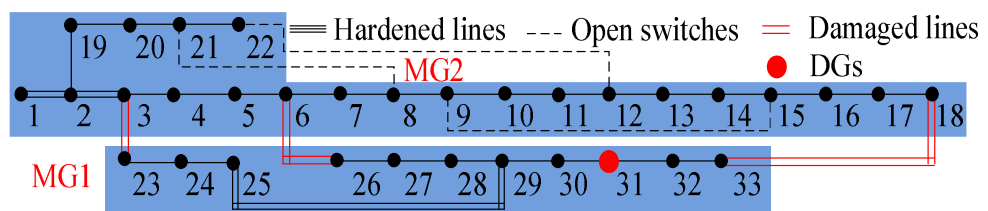

Fig.11 Planning and operational strategies in 33-node system

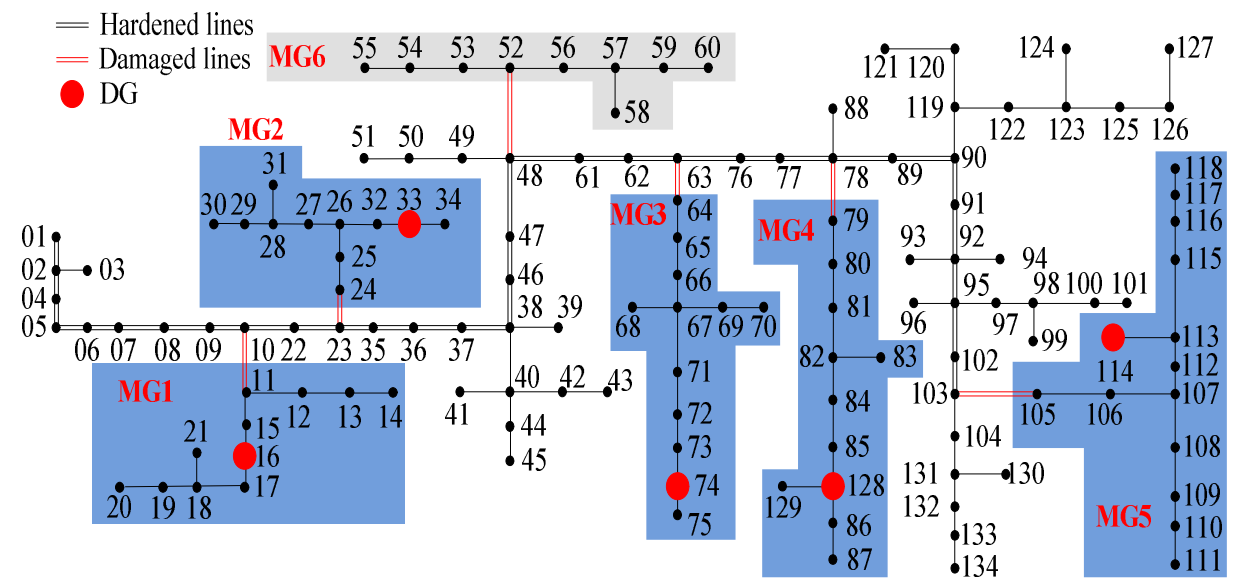

Fig.12 Planning and operational strategies in 135-node system 


\section{2) Effectiveness of the linearized DistFlow model}

In this section, the effectiveness of the linearized DistFlow model is validated by the comparative simulations with the AC model. In the 13-node system, it is assumed the lines 13 and 15 are damaged by the natural disaster. In the 33-node and 135-node system, the damaged lines are shown in Fig.11 and Fig.12. In this case, the power loss and load shedding obtained from the linearized DistFlow model and the AC model with the planning decisions are shown in Table X. Moreover, the gap of the voltage amplitude between the linearized DistFlow model and the AC model is shown in Fig.13-a, Fig.13-b, and Fig.13-c. It can be seen that, although some simplification techniques are applied in the linearized DistFlow model, such as ignoring the power loss, the precision of the linearized DistFlow model can be acceptable. Specifically, the load shedding in the linearized DistFlow model, which is our focus to reduce, is almost same with that in the AC model. Moreover, the maximum difference of the voltage amplitude between the linearized DistFlow model and the AC model is $3 \times 10^{-3}$ p.u., and this difference has little effect on the precision of the proposed model. Consequently, according to the comparative simulations with the $\mathrm{AC}$ model, the precision of the linearized DistFlow model can be sufficient for the planning problem in this paper.

TABLE X COMPARISON BETWEEN THE LINEARIZED DISTFLOW MODEL AND THE AC MODEL

\begin{tabular}{|c|c|c|c|c|}
\hline \multirow{2}{*}{ System } & \multicolumn{2}{|c|}{ The percentage of the power loss to loads (\%) } & \multicolumn{2}{|c|}{ The load shedding $(\mathrm{kWh})$} \\
\hline & The linearized Distflow method & The AC method & The linearized Distflow method & The AC method \\
\hline 13-node & 0 & 0.28 & 5704 & 5704 \\
\hline 33-node & 0 & 1.27 & 34800 & 34802 \\
\hline 135-node & 0 & 1.33 & 64817 & 64884 \\
\hline
\end{tabular}

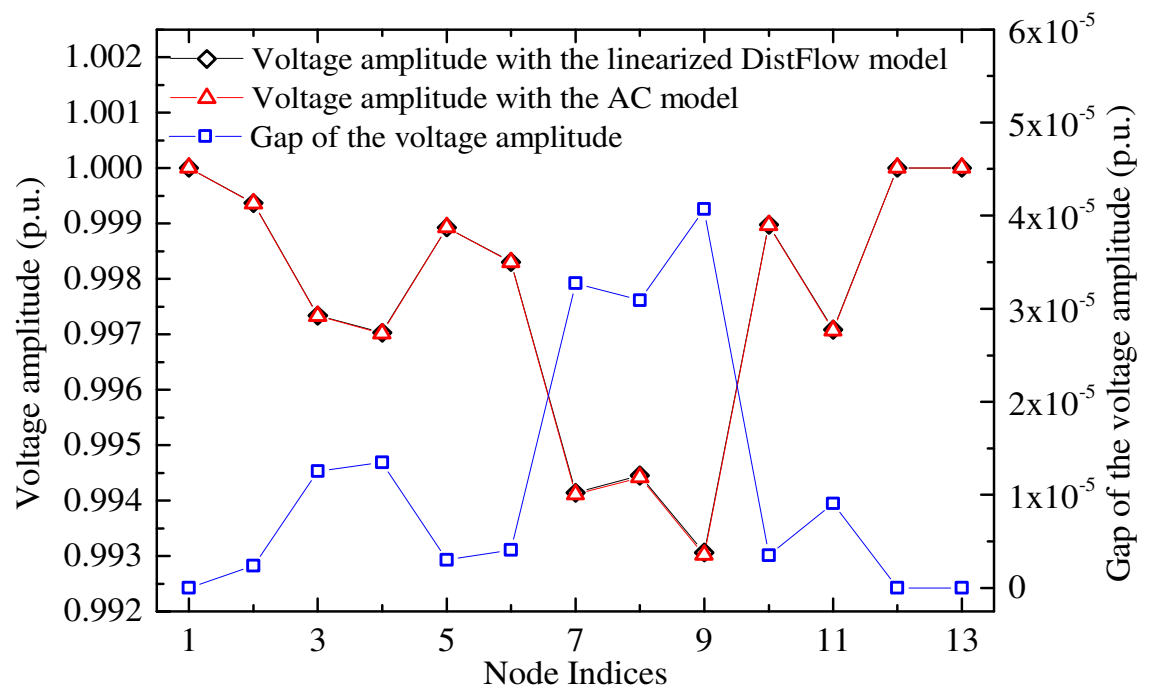

Fig.13-a Gap of the voltage amplitude in 13-node system 


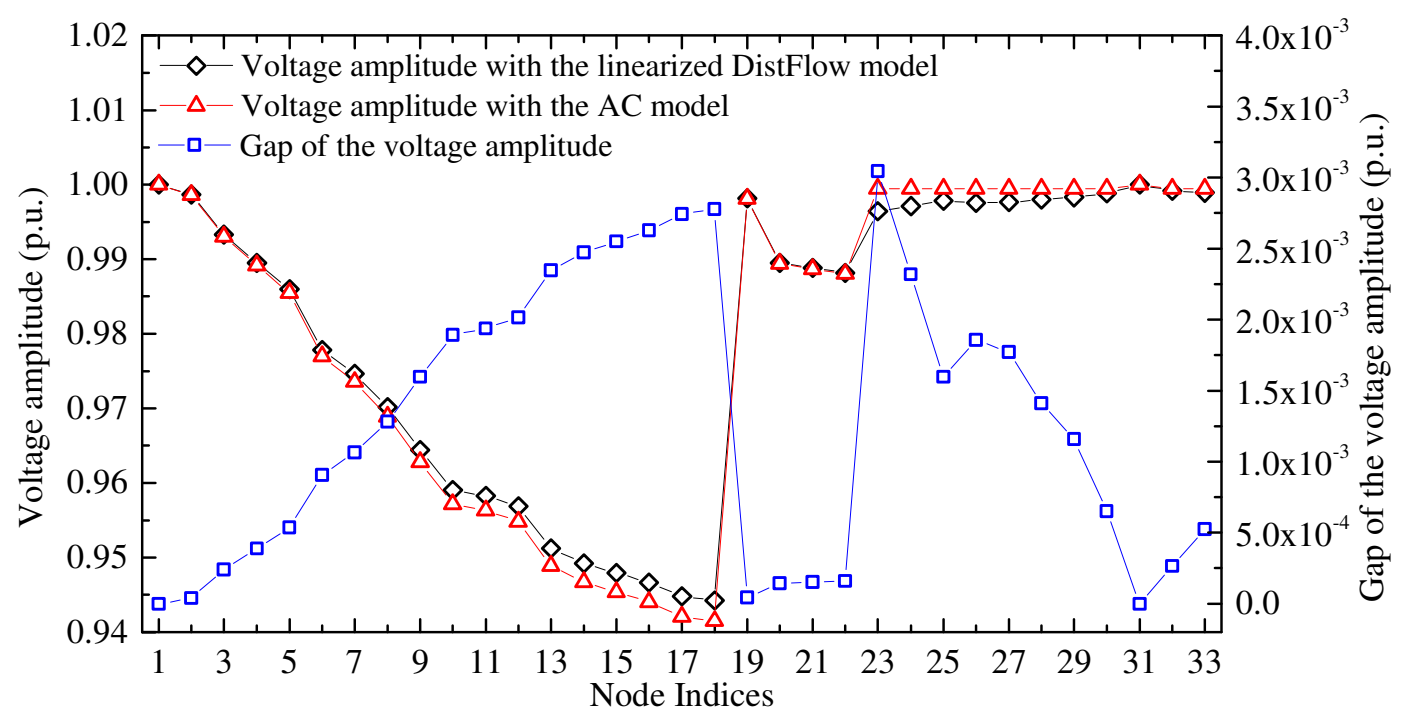

Fig.13-b Gap of the voltage amplitude in 33-node system

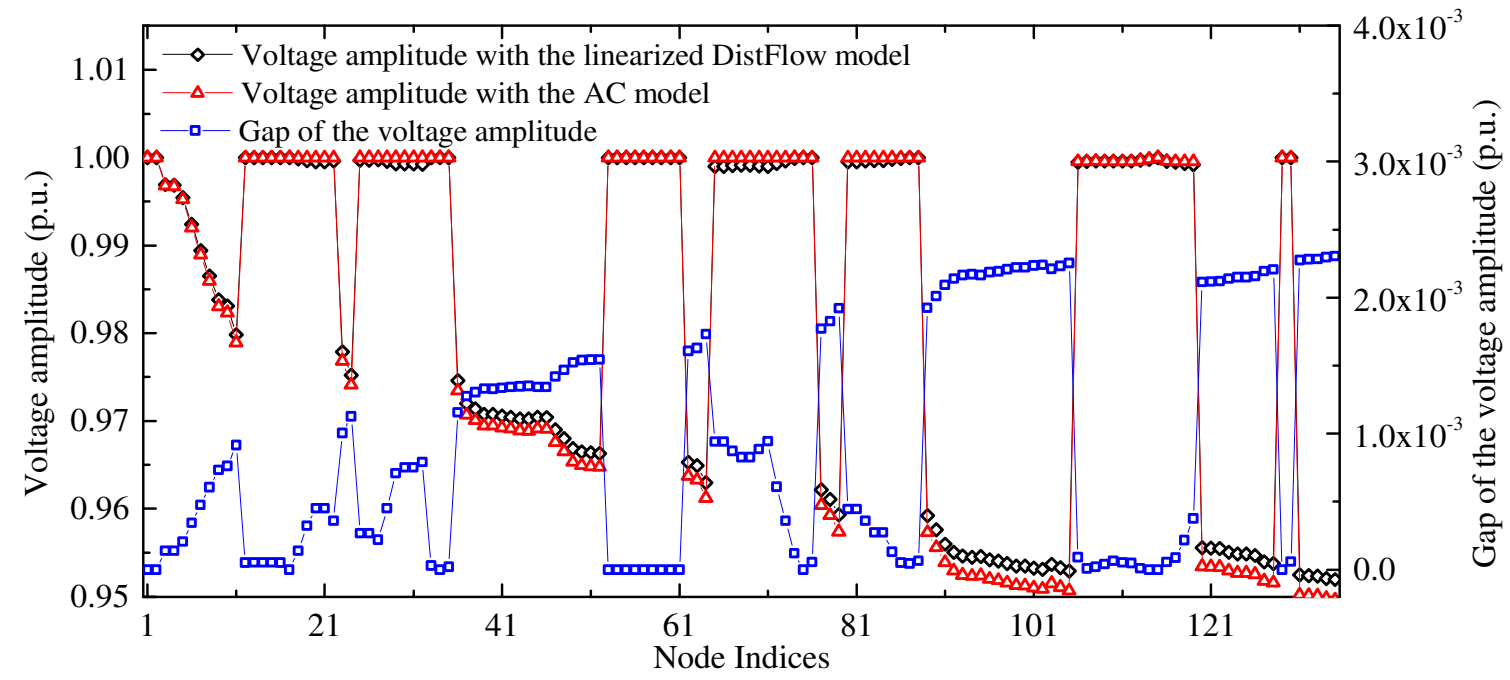

Fig.13-c Gap of the voltage amplitude in 13-node system

\section{3) Effectiveness of the solution method}

In this section, the effectiveness of the solution method is demonstrated. The gap is set to be $10^{-5}$. The value of $L B$ and $U B$ in Case 1, Case 9 and Case 10 is shown in Fig.14-a, Fig.14$\mathrm{b}$ and Fig.14-c, respectively. It can be seen that the primal cut based solution method and dynamically refine the value of LB and UB, thereby generating an optimal solution. Moreover, the system size has little effect on the iteration number of the solution method, hence the effectiveness of the solution method to the large systems can be guaranteed. Then, the performance of the CCG method is evaluated, as shown in Fig. 15-a for Case1 s1, and Fig. 15-b for Case $1 \mathbf{s} 2$. It is noted that the CCG algorithm is implemented at each iteration of the primal cut based solution method. Hence, the times of the CCG implementation are identical to the iteration number of the primal cut based solution method. It can be seen from 
Fig. 15-a and Fig. 15-b that, the CCG method can converge to the optimal solution within 5 iterations for all cases, which indicates the effectiveness of the CCG method for the model (11).

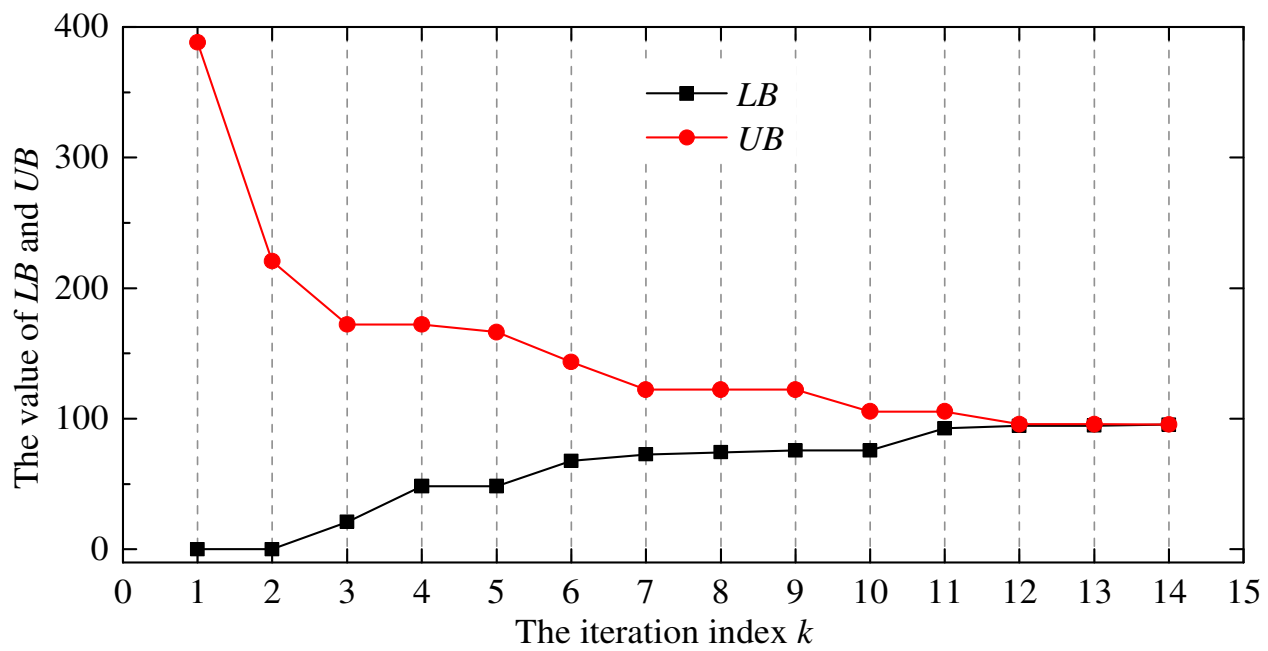

Fig.14-a The evolution of the value of $L B$ and $U B$ in case 1 for 13-node system

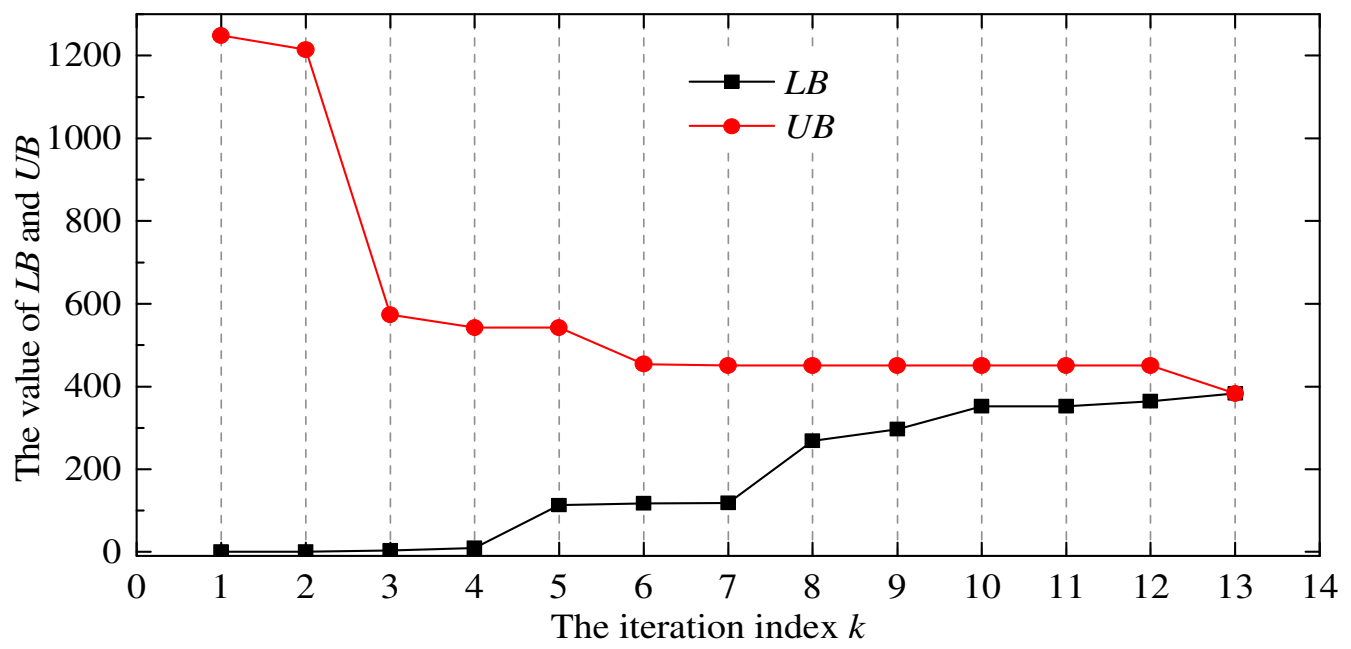

Fig.14-b The evolution of the value of $L B$ and $U B$ in case 9 for 33-node system 


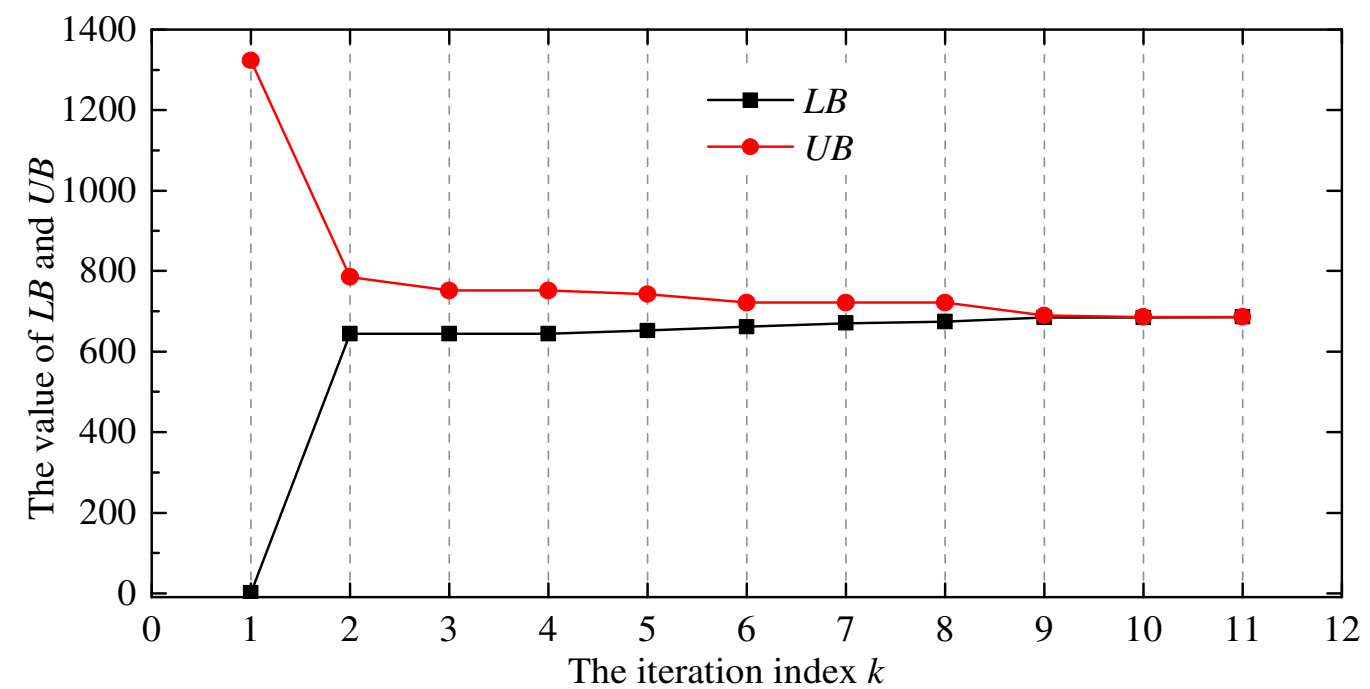

Fig.14-c The evolution of the value of $L B$ and $U B$ in case 10 for 135-node system

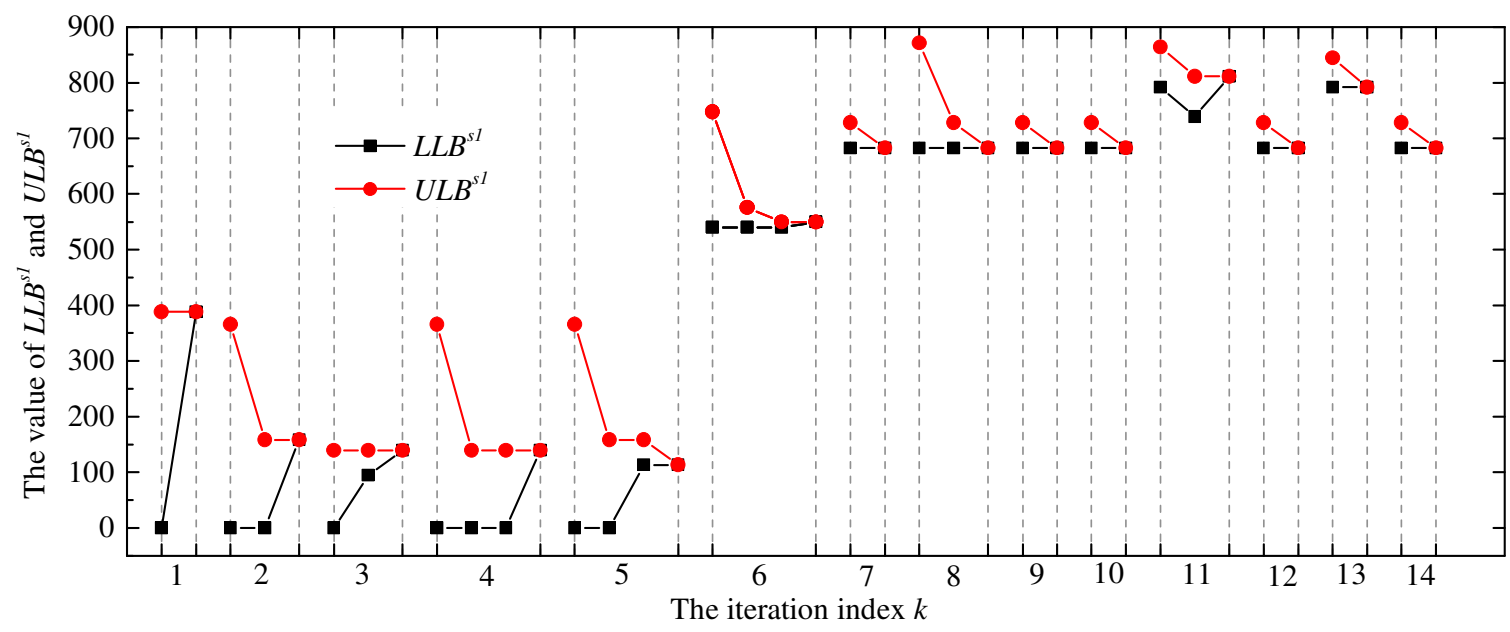

Fig.15-a The performance of the CCG method in Case 1 s1

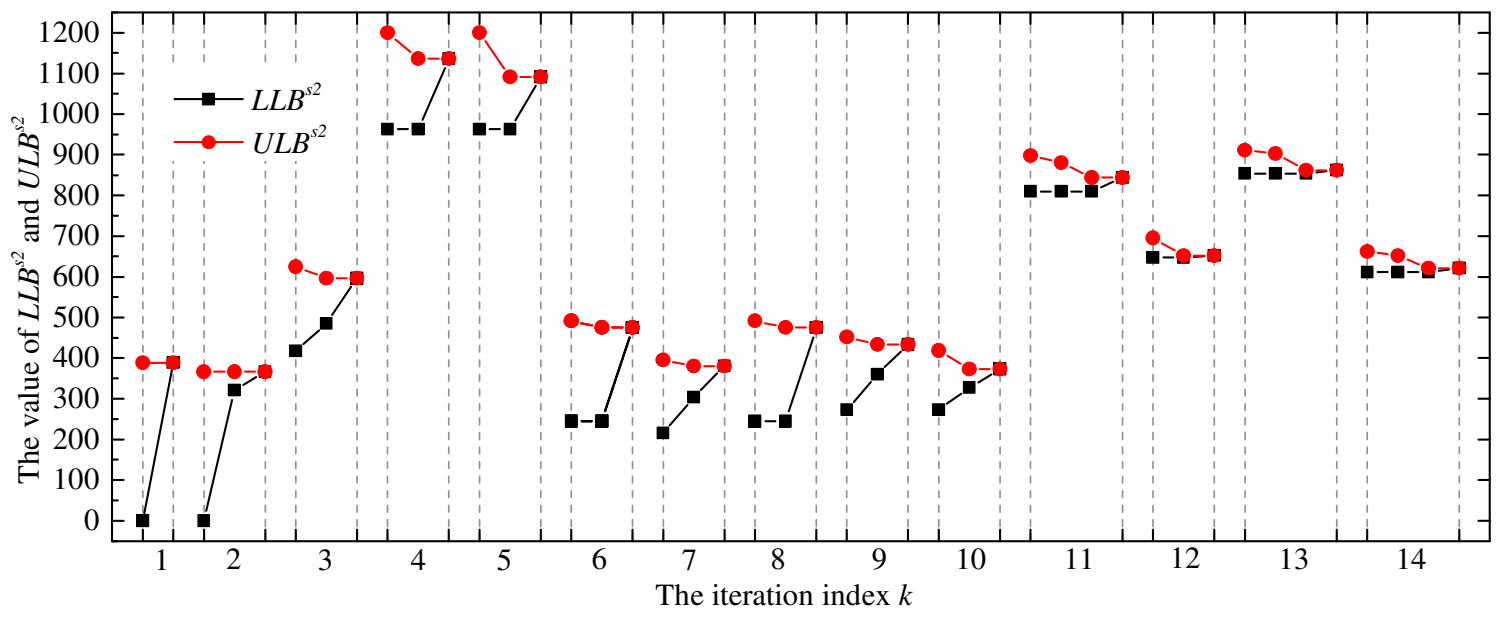

Fig.15-b The performance of the CCG method in Case 1 s 2 


\section{Conclusion}

This paper proposes a novel resilience-oriented planning model which is based on multidisaster-scenario and distributionally robust model (MDS-DRM) for line hardening and DG allocation in distribution systems. The consideration of the OR uncertainty with the stochastic method makes the planning strategy more robust against various natural disasters. The consideration of the PDLO uncertainty enables the planning model to address the random and nonlinear effect of the natural disasters to distribution systems. Compared with the existing methods, the proposed MDS-DRM can hedge against natural disasters in a more effective and less conservative manner. Besides, a novel primal cut based decomposition method is proposed to solve the multi-scenario two-stage distributionally robust programming model. The proposed solution method can converge to an optimal solution within finite iterations in all test distribution systems. In practical, the proposed method can be utilized to advise planning decisions to safeguard distribution systems from natural disasters.

\section{Appendix}

A. Derivation procedure from (7) to (8)

It is noted that $Z^{s}$ is formulated as a discrete and finite set containing all outage scenarios. In this case, the integral forms in (7) can be reformulated as the summation forms:

$$
\begin{aligned}
& \max _{\mathrm{P}^{s}} \int_{Z^{s}} Q\left(\mathbf{y}, \mathbf{z}^{s}\right) \mathrm{P}^{s}\left(\mathrm{~d} \mathbf{z}^{s}\right)=\sum_{\mathbf{z}^{s} \in Z^{s}} Q\left(\mathbf{y}, \mathbf{z}^{s}\right) \mathrm{gP}^{s}\left(\mathbf{z}^{s}\right) \\
& \int_{Z^{s}} \mathbf{B} \mathbf{z}^{s} \mathrm{P}^{s}\left(\mathrm{~d} \mathbf{z}^{s}\right)=\sum_{\mathbf{z}^{s} \in Z^{s}} \mathbf{B} \mathbf{z}^{s} \mathrm{gP}^{s}\left(\mathbf{z}^{s}\right) \\
& \int_{Z^{s}} \mathrm{P}^{s}\left(\mathrm{~d} \mathbf{z}^{s}\right)=\sum_{\mathbf{z}^{s} \in Z^{s}} \mathrm{P}^{s}\left(\mathbf{z}^{s}\right)
\end{aligned}
$$

Then, the summation form of (7) can be obtained by integrating (A1), (A2) and (A3) into (7) as follows, where the decision variable is the probability of all outage scenarios.

$$
\begin{array}{lll}
\max _{\mathrm{P}^{s}} & \sum_{\mathbf{z}^{s} \in Z^{s}} Q\left(\mathbf{y}, \mathbf{z}^{s}\right) \mathrm{gP}^{s}\left(\mathbf{z}^{s}\right) \\
\text { s.t. } & \sum_{\mathbf{z}^{s} \in Z^{s}} \mathbf{B z}^{s} \mathrm{gP}^{s}\left(\mathbf{z}^{s}\right) \leq \mathbf{c}^{s} \quad: \quad \boldsymbol{\alpha}^{s} \\
& \sum_{\mathbf{z}^{s} \in Z^{s}} \mathrm{P}^{s}\left(\mathbf{z}^{s}\right)=1 \quad: \quad \beta^{s}
\end{array}
$$

It is noted that the decision variable in the linear programming (A4) is the occurrence probability of all outage scenarios in $Z^{s}$, which is continuous variable ranging from 0 to 1 . Hence, (A4) can be transformed to the dualization form (A5) based on the standard duality.

$$
\begin{aligned}
& \min _{\boldsymbol{\alpha}^{s} \geq 0, \beta^{s}}\left(\mathbf{c}^{s}\right)^{\cdot} \cdot \boldsymbol{\alpha}^{s}+\beta^{s} \\
& \text { s.t. } \quad\left(\boldsymbol{\alpha}^{s}\right)^{\cdot} \mathbf{B} \mathbf{z}^{s}+\beta^{s} \geq Q\left(\mathbf{y}, \mathbf{z}^{s}\right) \quad \forall \mathbf{z}^{s} \in Z^{s}
\end{aligned}
$$




\section{$B$. The model for calculating the performance indicators}

The proposed MDS-DRM wishes to minimize the expected cost over all possible ORs. According to the definition in [42], [43], the proposed MDS-DRM is actually the recourse problem, and the optimal solution is called the recourse problem solution (RP).

If we could wait and see what the random OR would occur before we make the planning decision, then we could choose $\mathbf{y}^{s}$ for the specific realization of the OR uncertainty. Then, the wait-and-see solution (WS) can be defined as:

$$
\begin{array}{ll}
\mathrm{WS}= & \sum_{s \in S} \rho^{s} \cdot\left\{\min \mathbf{a} \mathbf{y}^{s}+\sup _{\mathrm{P}^{s} \in \mathrm{F}^{s}} \mathrm{E}_{\mathrm{P}^{s}}\left[Q\left(\mathbf{y}^{s}, \mathbf{z}^{s}\right)\right]\right\} \\
\text { s.t. } \quad & \mathbf{A y} \mathbf{y}^{s} \leq \mathbf{b}, \quad \forall s \in S \\
& \mathrm{~F}^{s}=\left\{\mathrm{P}^{s} \in \mathrm{P}^{s}\left(Z^{s}\right): \mathrm{E}_{\mathrm{P}^{s}}\left[\mathbf{B} \mathbf{z}^{s}\right] \leq \mathbf{c}^{s}\right\} \forall s \in S \\
& \left\{\begin{array}{l}
Q\left(\mathbf{y}^{s}, \mathbf{z}^{s}\right)=\min _{\mathbf{x}^{s}, \mathbf{v}^{s}} \mathbf{d}^{\mathrm{T}} \mathbf{x}^{s} \quad \forall s \in S \\
\mathbf{C} \mathbf{y}^{s}+\mathbf{D} \mathbf{z}^{s}+\mathbf{E} \mathbf{x}^{s}+\mathbf{F} \mathbf{v}^{s} \leq \mathbf{e} \quad \forall s \in S
\end{array}\right.
\end{array}
$$

The expected value solution (EV) can be obtained by solving the expected value problem, where all OR-related random variables are replaced by their expected values, thereby solving a deterministic program:

$$
\begin{aligned}
\mathrm{EV}= & \min \mathbf{a}^{\cdot} \mathbf{y}+\sup _{\mathrm{P} \in \mathrm{F}} \mathrm{E}_{\mathrm{P}}[Q(\mathbf{y}, \mathbf{z})] \\
\text { s.t. } \quad & \mathbf{A y} \leq \mathbf{b} \\
& \mathrm{F}=\left\{\mathrm{P} \in \mathrm{P}(Z): \mathrm{E}_{\overline{\mathrm{P}}}[\mathbf{B z}] \leq \mathrm{E}_{s \in S}\left(\mathbf{c}^{s}\right), \quad \mathrm{E}_{s \in S}\left(\mathbf{c}^{s}\right)=\sum_{s \in S} \rho^{s} \cdot \mathbf{c}^{s}\right\} \\
& \left\{\begin{array}{l}
Q(\mathbf{y}, \mathbf{z})=\min _{\mathbf{x}, \mathbf{v}} \mathbf{d}^{\mathrm{T}} \mathbf{x} \\
\mathbf{C y}+\mathbf{D z}+\mathbf{E x}+\mathbf{F v} \leq \mathbf{e}
\end{array}\right.
\end{aligned}
$$

Let $\overline{\mathbf{y}}$ be an optimal solution to (B2), called the expected value solution. The expected value of using the EV solution (EEV) can be obtained by:

$$
\mathrm{EEV}=\min \mathbf{a}^{\cdot} \overline{\mathbf{y}}+\sum_{s \in S} \rho^{s} \cdot \sup _{\mathrm{P}^{s} \in \mathrm{F}^{s}} \mathrm{E}_{\mathrm{P}^{s}}\left[Q\left(\mathbf{y}, \mathbf{z}^{s}\right)\right]
$$

s.t. Constraints $(6 \mathrm{c}),(6 \mathrm{~d})$

\section{The proof for the Guaranteed Convergence}

It is first claimed that any repeated $\mathbf{z}^{s, \omega}$ in the solution procedure implies the optimality of (9), i.e., $L B^{\omega}=U B^{\omega}$. Then, $Z^{s}$ in (2) is a finite set. 
Suppose at iteration $\omega, \mathbf{z}^{s, \omega}$ can be obtained from (11), which leads to optimal $\mathbf{x}^{s, \omega}$ and $O b j L^{s, \omega}$. It follows that:

$O b j L^{s, \omega}=\mathbf{d}^{\mathrm{T}} \mathbf{x}^{s, \omega}-\left(\boldsymbol{\alpha}^{s, \omega}\right)^{\bullet} \mathbf{B} \mathbf{z}^{s, \omega} \leq \mathbf{d}^{\mathrm{T}} \mathbf{x}^{s}-\left(\boldsymbol{\alpha}^{s, \omega}\right)^{\bullet} \mathbf{B} \mathbf{z}^{s, \omega}, \forall \mathbf{x}^{s}$

According to (12), it can be obtained that:

$$
\begin{aligned}
U B^{\omega} & \leq O b j U^{\omega}-\sum_{s \in S} \rho^{s} \cdot \beta^{s, \omega}+\sum_{s \in S} \rho^{s} \cdot O b j L^{s, \omega} \\
& =\mathbf{a}^{\cdot} \mathbf{y}^{\omega}+\sum_{s \in S} \rho^{s} \cdot\left(\mathbf{c}^{s}\right)^{\bullet} \cdot \boldsymbol{\alpha}^{s, \omega}+\sum_{s \in S} \rho^{s} \cdot \operatorname{Obj} L^{s, \omega}
\end{aligned}
$$

If $\mathbf{z}^{s, \omega}$ has been identified in a previous iteration $\omega^{s}$ with $1 \leq \omega^{s} \leq \omega$, then according to the primal cut 1 in (10), it can be obtained that $\beta^{s, \omega} \geq O \operatorname{Obj} L^{s, \omega}$ by:

$$
\beta^{s, \omega} \geq \mathbf{d}^{\mathrm{T}} \mathbf{x}^{s}-\left(\boldsymbol{\alpha}^{s}\right)^{\bullet} \mathbf{B} \mathbf{z}^{s, \omega_{s}}=\mathbf{d}^{\mathrm{T}} \mathbf{x}^{s}-\left(\boldsymbol{\alpha}^{s}\right)^{\bullet} \mathbf{B} \mathbf{z}^{s, \omega} \geq \mathbf{d}^{\mathrm{T}} \mathbf{x}^{s, \omega}-\left(\boldsymbol{\alpha}^{s, \omega}\right)^{\bullet} \mathbf{B} \mathbf{z}^{s, \omega}=O \operatorname{Obj} L^{s, \omega}
$$

Hence, according to the model (10), it can be derived that:

$$
\begin{aligned}
L B^{\omega}=O b j U^{\omega} & =\mathbf{a}^{\cdot} \mathbf{y}^{\omega}+\sum_{s \in S} \rho^{s} \cdot\left[\left(\mathbf{c}^{s}\right)^{\bullet} \cdot \boldsymbol{\alpha}^{s, \omega}+\beta^{s, \omega}\right] \\
& \geq \mathbf{a}^{\cdot} \mathbf{y}^{\omega}+\sum_{s \in S} \rho^{s} \cdot\left(\mathbf{c}^{s}\right)^{\cdot} \cdot \boldsymbol{\alpha}^{s, \omega}+\sum_{s \in S} \rho^{s} \cdot \operatorname{Obj} L^{s, \omega}
\end{aligned}
$$

Then, based on (C2), (C4), and the fact that $U B^{\omega} \geqslant L B^{\omega}$, it follows that $U B^{\omega}=L B^{\omega}$. The proof of convergence is completed.

\section{Reference}

[1] Li G, Zhang P, Luh PB, Li W, Bie Z, Serna C, et al. Risk analysis for distribution systems in the northeast U.S. under wind storms. IEEE Trans Power Syst 2014;29(2):889-898. http://dx.doi.org/10.1109/TPWRS.2013.2286171

[2] C. Chen, J. Wang and D. Ton, Modernizing distribution system restoration to achieve grid resiliency against extreme weather events: An integrated solution, Proc. IEEE 2017;105(7):1267-1288 https://doi.org/10.1109/JPROC.2017.2684780

[3] Y. Xu, C. Liu, K. P. Schneider, and D. T. Ton. Toward a resilient distribution system. Proc. IEEE Power Energy Soc. Gen. Meeting, Denver, CO, USA, 2015: $1-5$. https://doi.org/10.1109/PESGM.2015.7286551

[4] M. A. Mohamed, T. Chen, W. Su and T. Jin. Proactive resilience of power systems against natural disasters: A literature review. IEEE Access 2019;7:163778-163795. https://doi.org/10.1109/ACCESS.2019.2952362

[5] G. Muñoz-Delgado, J. Contreras and J. M. Arroyo. Joint expansion planning of distributed generation and distribution networks. IEEE Trans
Power Syst 2015;30(5):2579-2590. https://doi.org/10.1109/TPWRS.2014.2364960

[6] G. Muñoz-Delgado, J. Contreras and J. M. Arroyo. Distribution system expansion planning considering non-utility-owned DG and an independent distribution system operator. IEEE Trans Power Syst 2019;34(4):2588-2597. https://doi.org/10.1109/TPWRS.2019.2897869

[7] G. Muñoz-Delgado, J. Contreras and J. M. Arroyo. Multistage generation and network expansion planning in distribution systems considering uncertainty and reliability. IEEE Trans Power Syst 2016;31(5):3715-3728. https://doi.org/10.1109/TPWRS.2015.2503604

[8] G. Muñoz-Delgado, J. Contreras and J. M. Arroyo. Distribution network expansion planning with an explicit formulation for reliability assessment. IEEE Trans Power Syst 2018;33(3):2583-2596. https://doi.org/10.1109/TPWRS.2017.2764331

[9] P. Dehghanian, S. Aslan and P. Dehghanian. Maintaining electric system safety through an enhanced network resilience. IEEE Trans. Industry Applications 2018;54(5):4927-4937. https://doi.org/10.1109/TIA.2018.2828389 
[10] C. Chen, J. Wang, F. Qiu and D. Zhao. Resilient distribution system by microgrids formation after natural disasters. IEEE Trans. Smart Grid 2016;7(2):958-966. https://doi.org/10.1109/TSG.2015.2429653

[11] Z. Bie, Y. Lin, G. Li and F. Li. Battling the extreme: A study on the power system resilience. Proc. IEEE 2017;105(7):1253-1266. https://doi.org/ 10.1109/JPROC.2017.2679040

[12] T. Ding, Y. Lin, Z. Bie and C. Chen. A resilient microgrid formation strategy for load restoration considering master-slave distributed generators and topology reconfiguration. Appl. Energy 2017:199:205-216. https://doi.org/10.1016/j.apenergy.2017.05.012

[13] S. Xie, Z. Hu, D. Zhou, Y. Li, S. Kong, W. Lin, Y. Zheng. Multi-objective active distribution networks expansion planning by scenario-based stochastic programming considering uncertain and random weight of network. Appl. Energy 2018; 219:207-225. https://doi.org/10.1016/j.apenergy.2018.03.023.

[14] S. Ma, L. Su, Z. Wang, F. Qiu and G. Guo. Resilience enhancement of distribution grids against extreme weather events. IEEE Trans Power Syst 2018;33(5):4842-4853. https://doi.org/10.1109/TPWRS.2018.2822295

[15] S. Ma, S. Li, Z. Wang and F. Qiu. Resilienceoriented design of distribution systems. IEEE Trans Power Syst 2019;34(4):2880-2891. https://doi.org/10.1109/TPWRS.2019.2894103

[16] J. Kim and Y. Dvorkin. Enhancing distribution system resilience with mobile energy storage and microgrids. IEEE Trans. Smart Grid 2019;10(5):4996-

5006. https://doi.org/10.1109/TSG.2018.287252 1

[17] S. Lei, J. Wang, C. Chen and Y. Hou. Mobile emergency generator pre-positioning and realtime allocation for resilient response to natural disasters. IEEE Trans. Smart Grid 2018;9(3):2030-2041. https://doi.org/10.1109/TSG.2016.2605692

[18]R. Jiang, J. Wang and Y. Guan. Robust unit commitment with wind power and pumped storage hydro. IEEE Trans. Power Syst 2012;27(2): 800-810. https://doi.org/10.1109/TPWRS.2011.2169817

[19]B. $\mathrm{Hu}, \mathrm{L}$. Wu and M. Marwali. On the robust solution to SCUC with load and wind uncertainty correlations. IEEE Trans. Power Syst 2019;29(6):2952-2964.

https://doi.org/10.1109/TPWRS.2014.2308637
[20] S. Xie, Z. Hu, J. Wang. Two-stage robust optimization for expansion planning of active distribution systems coupled with urban transportation networks. Appl Energy 2020;261:114412. https://doi.org/10.1016/j.apenergy.2019.114412

[21] M. Wu, L. Kou, X. Hou, Y. Ji, B. Xu, H. Gao. A bi-level robust planning model for active distribution networks considering uncertainties of renewable energies. Int J of Electr Power Energy Syst 2019;105:814-822. https://doi.org/10.1016/j.ijepes.2018.09.032

[22] X. Wang, Z. Li, M. Shahidehpour and C. Jiang. Robust line hardening strategies for improving the resilience of distribution systems with variable renewable resources. IEEE Trans. Sustain. Energy 2019;10(1):386-395.

https://doi.org/10.1109/TSTE.2017.2788041

[23] Y. Lin and Z. Bie. Tri-level optimal hardening plan for a resilient distribution system considering reconfiguration and DG islanding. Appl. Energy 2019;210:1266-1279. https://doi.org/10.1016/j.apenergy.2017.06.059

[24] N. Alguacil, A. Delgadillo and J. M. Arroyo. A trilevel programming approach for electric grid defense planning. Comput. Oper Res 2014;41: 282-290.

https://doi.org/10.1016/j.cor.2013.06.009

[25] W. Yuan, L. Zhao and B. Zeng. Optimal power grid protection through a defender-attackerdefender model. Rel. Eng. Syst. Safety 2014;121: 83-89. https://doi.org/10.1016/j.ress.2013.08.003

[26] W. Yuan, J. Wang, F. Qiu, C. Chen, C. Kang and B. Zeng. Robust optimization-based resilient distribution network planning against natural disasters. IEEE Trans. Smart Grid 2016;7(6):110. https://doi.org/10.1109/TSG.2015.2513048

[27] S. Ma, B. Chen and Z. Wang. Resilience enhancement strategy for distribution systems under extreme weather events. IEEE Trans. Smart Grid 2018;9(2):1442-1451. https://doi.org/10.1109/TSG.2016.2591885

[28] S. Babaei, C. Zhao and T. Ding. Allocating distributed generators for resilient distribution system under uncertain probability distribution of natural disasters. Power \& Energy Society General Meeting, Portland, OR USA, 2018:1-5. https://doi.org/10.1109/PESGM.2017.8274133

[29] Y. Xiang and L. Wang. An improved defenderattacker-defender model for transmission line defense considering offensive resource uncertainties. IEEE Trans. Smart Grid 2019;10(3):2534-2546. https://doi.org/ 10.1109/TSG.2018.2803783 
[30]C. Zhao and R. Jiang. Distributionally robust contingency-constrained unit commitment. IEEE Trans. Power Syst 2018;33(1): 94-102. https://doi.org/ 10.1109/TPWRS.2017.2699121

[31]P. Xiong, P. Jirutitijaroen and C. Singh. A distributionally robust optimization model for unit commitment considering uncertain wind power generation. IEEE Trans. Power Syst 2017;32(1):39-49. https://doi.org/10.1109/TPWRS.2017.2699121

[32]M. E. Baran and F. F. Wu. Network reconfiguration in distribution systems for loss reduction and load balancing IEEE Trans. Power Del 1989;4(2):1401-1407. https://doi.org/10.1109/61.25627

[33] Balakrishnan R and Ranganathan K. A Textbook of Graph Theory. New York, NY: Springer New York; 2012. https://doi.org/10.1007/978-1-46144529-6.

[34] H. Gao, Y. Chen, Y. Xu and C. Liu. Resilienceoriented critical load restoration using microgrids in distribution systems. IEEE Trans Smart Grid 2016;7(6):2837-2848. https://doi.org/10.1109/TSG.2016.2550625

[35]Z. Wang, C. Shen, Y. Xu, F. Liu, X. Wu and C. Liu. Risk-limiting load restoration for resilience enhancement with intermittent energy resources. IEEE Trans Smart Grid2019;10(3):2507-2522. https://doi.org/ 10.1109/TSG.2018.2803141

[36] Tao Ding, Yanling Lin, Zhaohong Bie, Chen Chen. A resilient microgrid formation strategy for load restoration considering master-slave distributed generators and topology reconfiguration. Appl Energy 2017;199:205-216. https://doi.org/10.1016/j.apenergy.2017.05.012.
[37] B. Zeng and L. Zhao. Solving two-stage robust optimization problems using a column-andconstraint generation method. Oper. Res. Lett., 2013;41(5):457-461.

https://doi.org/10.1016/j.orl.2013.05.003

[38] L. Zhao and B. Zeng. An exact algorithm for two-stage robust optimization with mixed integer recourse problems. 2012. [Online]. http://www.optimizationonline.org/DB_FILE/2012/01/3310.pdf

[39] H. Yuan, W. Zhang, J. Zhu, Amvrossios C. Bagtzoglou. Resilience assessment of overhead power distribution systems under strong winds for hardening prioritization. ASCE-ASME J Risk Uncertainty Engineer Syst, Part A: Civil Engineering 2018;4(4). https://doi.org/10.1061/AJRUA6.0000988

[40] J. Z. Zhu. Optimal reconfiguration of electrical distribution network using the refined genetic algorithm. Elect. Power Syst. Res 2002;62:37-42. https://doi.org/10.1016/S0378-7796(02)00041-X

[41]B. Pereira Jr., M. A. Cossi, and J. R. S. Mantovani. Multiobjective short-term planning of electric power distribution systems using NSGA-II. J. Control. Autom. Elect. Syst. 2013;14:1-12. https://doi.org/10.1007/s40313013-0022-5

[42] M. Avriel and A. C. Williams. The value of information and stochastic programming. Oper Res 1970;18(5):947-954. https://doi.org/10.1287/opre.18.5.947

[43] Maggioni, F., Wallace, S.W. Analyzing the quality of the expected value solution in stochastic programming. Ann Oper Res 2012;200:37-54. https://doi.org/10.1007/s10479010-0807-x 